Published in: Integral Equations and Operator Theory (IEOT), 76, 2013, 509-547,

DOI $10.1007 / \mathrm{s} 00020-013-2054-4$

\title{
Localized Boundary-Domain Singular Integral Equations Based on Harmonic Parametrix for Divergence-Form Elliptic PDEs with Variable Matrix Coefficients
}

\author{
O. Chkadua, S.E. Mikhailov and D. Natroshvili
}

\begin{abstract}
Employing the localized integral potentials associated with the Laplace operator, the Dirichlet, Neumann and Robin boundary value problems for general variable-coefficient divergence-form second-order elliptic partial differential equations are reduced to some systems of localized boundary-domain singular integral equations. Equivalence of the integral equations systems to the original boundary value problems is proved. It is established that the corresponding localized boundarydomain integral operators belong to the Boutet de Monvel algebra of pseudo-differential operators. Applying the Vishik-Eskin theory based on the factorization method, the Fredholm properties and invertibility of the operators are proved in appropriate Sobolev spaces.
\end{abstract}

Mathematics Subject Classification (2010). 35J25, 31B10, 45K05, 45 A05.

Keywords. Partial differential equations, variable coefficients, boundary value problems, localized parametrix, localized potentials, localized boundary-domain integral equations, pseudo-differential equations.

\section{Introduction}

We consider the Dirichlet, Neumann and Robin boundary value problems (BVPs) in a bounded domain for scalar second order divergent-form elliptic partial differential equations (PDEs) with variable matrix coefficients and develop the method of localized boundary-domain singular integral equations (LBDSIEs) based on a localized harmonic parametrix.

The basic boundary value problems treated in the paper are well investigated in the literature by the variational methods, and (when the corresponding fundamental solution is available in an explicit form) also by the classical potential methods, see, e.g., [18], [19], [20], [27], and the references 
therein. In the latter case, the BVPs in the domain can be reduced to boundary integral equations on the domain boundary, which can be used not only for the BVP analysis but also for effective numerical solution.

However, the fundamental solutions for second order elliptic partial differential equations with variable coefficients are not available in explicit form, in general. Application of the potential method based on the corresponding Levi function, which can be constructed explicitly, leads to Fredholm-Riesz type integral equations but invertibility of the integral operators was proved only for some particular cases (see [27] and the references therein).

For a mixed BVP for an elliptic PDE with a scalar variable coefficient in a three-dimensional bounded domain, an analysis (including invertibility) in Sobolev spaces of segregated direct boundary-domain integral equations (BDIEs) with a parametrix (Levi function) taken as a fundamental solution of a corresponding PDE with frozen coefficient, was developed in $[7,8]$ and extended to united direct boundary-domain integro-differential equations in [23]. For the same BVPs, two-operator segregated direct BDIEs, where a parametrix of another elliptic PDE was employed, were analysed in $[3,4]$. An analysis of segregated direct BDIEs for Dirichlet, Neumann and mixed BVPs for the PDE with variable scalar coefficient in a bounded domain with internal crack was presented in [10]. In [13], the direct segregated BDIEs for Dirichlet, Neumann and mixed BVPs in exterior (unbounded) domains have been investigated in weighted Sobolev spaces of the Beppo-Levi type.

The segregated direct localized boundary-domain integral equations (LBDIEs), introduced in [21] and associated with the Dirichlet and Neumann BVPs for the PDE with a scalar variable coefficient in a bounded threedimensional domain, were analysed in [9]. In [11] we considered segregated LBDIEs for an elliptic second order PDE with a scalar variable coefficient in a composite domain under the transmission conditions on the interface and the Dirichlet or mixed boundary conditions on the external boundary. In [12] the latter analysis was extended to segregated LBDIEs associated with the Dirichlet, Neumann and mixed BVPs in a composite domain with a crack on the interface, for an elliptic second order scalar PDE with variable matrix coefficients of a particular form, where the PDE matrix coefficients in adjacent regions of the composite domain are representable as the product of constant matrices and scalar functions. In the references [9], [11] and [12], it was possible to represent the localised parametrix of the considered PDE as the product of a scalar function and the localised parametrix of a constantcoefficient partial differential operator. As a result, all the domain integral operators involved in the corresponding LBDIE systems were at most weekly singular, and the multiplicative form of the parametrix essentially facilitated the analysis.

However such possibility seems to be not available for scalar second order PDEs with general matrix variable coefficients. In this paper, our main goal is to show that employing the localized parametrix of the Laplace operator, the Dirichlet, Neumann and Robin BVPs for a scalar elliptic PDE with 
variable matrix coefficients of general form can be reduced to some systems of segregated direct localized boundary-domain singular integral equations. Following $[22,3,4]$, we call them two-operator LBDSIE systems, since they are obtained by applying a parametrix of the Laplace operator to solve BVPs for another scalar second order elliptic partial differential operator with variable coefficients, for which it is not a parametrix, in general. We prove that the corresponding localized boundary-domain singular (in the Cauchy sense) integral operators (LBDSIOs) are invertible in appropriate Sobolev spaces. Beside a pure mathematical interest, these results look also important for constructing LBDSIE-based effective numerical algorithms, cf. [32, 33, 31, 30, 21, 25].

The paper is organized as follows. In Section 2, we formulate the Dirichlet, Neumann, and Robin BVPs for an elliptic PDE with matrix variable coefficients in appropriate Sobolev spaces. Then we introduce the localized harmonic parametrix as the product of the fundamental solution function of the Laplace operator and an appropriately chosen localising function. The harmonic parametrix is neither a fundamental solution nor a parametrix of the variable-coefficient PDE under consideration, in general (unlike the scalarcoefficient case considered in [9]). Using the localized harmonic parametrix, we derive a two-operator localized parametrix-based version of the Green third identity for a second order divergent-form elliptic partial differential operator with variable coefficients and establish some auxiliary identities needed in our further analysis.

In Sections 3, with the help of Green's third identity derived in Section 2, we reformulate the Dirichlet, Neumann, and Robin BVPs as direct localized boundary-domain integral equations systems and prove the corresponding equivalence theorems.

In Sections 4, we investigate Fredholm properties of the domain singular integral operator which appears in the obtained LBDSIE systems.

In Sections 5, 6, and 7 we analyse in detail Fredholm properties of the LBDSIOs associated with the Dirichlet, Robin, and Neumann BVPs, respectively. We establish that the LBDSIOs belong to the Boutet de Monvel algebra of pseudo-differential operators [5, 17, 28] and employing the VishikEskin theory $[15,29]$ based on the factorization method we prove invertibility of the LBDSIOs in appropriate Sobolev spaces. The most essential point here is to show that the so called generalized Šapiro-Lopatinskii conditions hold, implying that the LBDSIOs under consideration are Fredholm. Afterwards we establish that the indices of the LBDSIOs equal to zero and characterise the corresponding null spaces. In particular, we show that the null spaces of the LBDSIOs associated with the Dirichlet and Robin problems are trivial which lead to invertibility of the LBDSIOs in appropriate Sobolev spaces, while the LBDSIO associated with the Neumann problem possesses a one-dimensional nontrivial null space.

To make the paper more self-contained, we describe shortly classes of localizing cut-off functions in Appendix A, while in Appendices B and $\mathrm{C}$ we 
collect some auxiliary results regarding the properties of localized potentials and the corresponding integral operators needed in our analysis.

\section{Boundary value problems and parametrix-based operators}

\subsection{Formulation of the problems and basic relations}

Consider a uniformly elliptic second order scalar partial differential operator in $\mathbb{R}^{3}$,

$$
A\left(x, \partial_{x}\right) u=\frac{\partial}{\partial x_{k}}\left(a_{k j}(x) \frac{\partial u}{\partial x_{j}}\right),
$$

where $\partial_{x}=\left(\partial_{1}, \partial_{2}, \partial_{3}\right), \partial_{j}=\partial_{x_{j}}=\partial / \partial x_{j}$, real coefficients $a_{k j} \in C^{\infty}\left(\mathbb{R}^{3}\right)$, and $a_{k j}=a_{j k}, j, k=1,2,3$. Here and in what follows we assume summation from 1 to 3 over repeated indices if not explicitly stated otherwise. Due to the uniform ellipticity, the matrix $\mathbf{a}(x):=\left[a_{k j}(x)\right]_{3 \times 3}$ is positive definite, i.e., there are positive constants $c_{1}$ and $c_{2}$ such that

$$
c_{1}|\xi|^{2} \leq A(x, \xi):=a_{k j}(x) \xi_{k} \xi_{j} \leq c_{2}|\xi|^{2} \quad \forall x \in \mathbb{R}^{3}, \quad \forall \xi \in \mathbb{R}^{3} .
$$

Further, let $\Omega^{+}$be an open bounded domain in $\mathbb{R}^{3}$ with a simply connected boundary $\partial \Omega^{+}=S \in C^{\infty}, \overline{\Omega^{+}}=\Omega^{+} \cup S$. Throughout the paper $n=\left(n_{1}, n_{2}, n_{3}\right)$ denotes the unit normal vector to $S$ directed outward the domain $\Omega^{+}$. Set $\Omega^{-}:=\mathbb{R}^{3} \backslash \overline{\Omega^{+}}$.

By $H^{r}(\Omega)=H_{2}^{r}(\Omega), H_{\mathrm{loc}}^{r}(\Omega)=H_{2, \mathrm{loc}}^{r}(\Omega), H_{\text {comp }}^{r}(\Omega)=H_{2, \text { comp }}^{r}(\Omega)$, and $H^{r}(S)=H_{2}^{r}(S), r \in \mathbb{R}$, we denote the Bessel potential spaces on a domain $\Omega$ and on a closed manifold $S$ without boundary, $\mathcal{D}(\Omega)$ stands for the space of infinitely differentiable test functions with compact support in $\Omega$, while $\mathcal{D}(\bar{\Omega})$ denotes the space of restrictions to $\bar{\Omega}$ of all functions from $\mathcal{D}\left(\mathbb{R}^{3}\right)$. Recall that $H^{0}(\Omega)=L_{2}(\Omega)$ is a space of square integrable functions in $\Omega$ and for $r \geqslant 0$ the Bessel potential space $H^{r}$ coincides with the SobolevSlobodetskii space $W_{2}^{r}$.

By $H^{1,0}\left(\Omega^{ \pm} ; A\right)$ we denote the following subspaces of $H^{1}\left(\Omega^{ \pm}\right)$, respectively,

$$
H^{1,0}\left(\Omega^{ \pm} ; A\right):=\left\{u \in H^{1}\left(\Omega^{ \pm}\right): A u \in H^{0}\left(\Omega^{ \pm}\right)\right\},
$$

endowed with the following graph norms

$$
\|u\|_{H^{1,0}\left(\Omega^{ \pm} ; A\right)}:=\left\{\|u\|_{H^{1}\left(\Omega^{ \pm}\right)}^{2}+\|A u\|_{H^{0}\left(\Omega^{ \pm}\right)}^{2}\right\}^{1 / 2} .
$$

Further, let $H_{\mathrm{loc}}^{1,0}\left(\Omega^{-} ; A\right):=\left\{u \in H_{\mathrm{loc}}^{1}\left(\Omega^{-}\right): A u \in H_{\mathrm{loc}}^{0}\left(\Omega^{-}\right)\right\}$.

By $\gamma^{-1}$ we denote a (non-unique) continuous linear extension operator acting from $H^{\frac{1}{2}}(S)$ into $H^{1}\left(\mathbb{R}^{3}\right)$. The restrictions of $\gamma^{-1}$ on $\Omega^{+}$and $\Omega^{-}$are the right inverse operators to the corresponding trace operators $\gamma^{+}$and $\gamma^{-}$.

The co-normal derivative operators on the surface $S$ for sufficiently smooth functions are defined by the relations

$$
T^{ \pm}\left(x, \partial_{x}\right) u(x):=a_{k j}(x) n_{k}(x) \gamma^{ \pm} \partial_{j} u(x), \quad x \in S .
$$


Hinted by Green's first identity, the co-normal derivative operator can be extended by continuity to $u \in H^{1,0}\left(\Omega^{+} ; A\right)$ as follows,

$$
\begin{array}{r}
\left\langle T^{+} u, g\right\rangle_{S}:=\int_{\Omega^{+}}\left(\gamma^{-1} g\right) A u d x+\int_{\Omega^{+}} a_{k j}(x)\left(\partial_{j} u\right)\left(\partial_{k} \gamma^{-1} g\right) d x \\
\forall g \in H^{\frac{1}{2}}(S),
\end{array}
$$

where $\langle\cdot, \cdot\rangle_{S}$ denotes the duality between the adjoint spaces $H^{-\frac{1}{2}}(S)$ and $H^{\frac{1}{2}}(S)$ which extends the usual bilinear $L_{2}(S)$ inner product. The generalized co-normal derivative operator $T^{-} u$ is defined similarly for functions $u \in H^{1,0}\left(\Omega^{-} ; A\right)$,

$$
\begin{array}{r}
\left\langle T^{-} u, g\right\rangle_{S}:=-\int_{\Omega^{-}}\left(\gamma^{-1} g\right) A u d x-\int_{\Omega^{-}} a_{k j}(x)\left(\partial_{j} u\right)\left(\partial_{k} \gamma^{-1} g\right) d x \\
\forall g \in H^{\frac{1}{2}}(S) .
\end{array}
$$

Definitions (2.3) and (2.4) do not depend on the extension operator $\gamma^{-1}$, cf. $[16,14,20,24]$. Hence, since $\gamma^{-1}$ can always be chosen such that $\gamma^{-1} g \in$ $H_{\text {comp }}^{1}\left(\mathbb{R}^{3}\right)$, the co-normal derivative operator $(2.4)$ is well defined on a wider class of functions $u \in H_{\mathrm{loc}}^{1,0}\left(\Omega^{-} ; A\right)$ for the unbounded domain $\Omega^{-}$.

By [14, Lemma 3.4], [20, Lemma 4.3]), the first Green identity holds for $u \in H^{1,0}(\Omega ; A)$ in the form

$$
\left\langle T^{+} u, \gamma^{+} v\right\rangle_{S}=\int_{\Omega^{+}}\left[v A u+a_{k j}(x)\left(\partial_{j} u\right)\left(\partial_{k} v\right)\right] d x \quad \forall v \in H^{1}(\Omega) .
$$

Then for arbitrary functions $u, v \in H^{1,0}(\Omega ; A)$ we have the second Green identity,

$$
\int_{\Omega^{+}}[v A u-u A v] d x=\left\langle T^{+} u, \gamma^{+} v\right\rangle_{S}-\left\langle T^{+} v, \gamma^{+} u\right\rangle_{S} .
$$
follows.

The Dirichlet, Neumann, and Robin boundary value problems read as

Dirichlet problem: Find a function $u \in H^{1,0}\left(\Omega^{+} ; A\right)$ satisfying the differential equation

$$
A\left(x, \partial_{x}\right) u=f \text { in } \Omega^{+}
$$

and the boundary condition

$$
\gamma^{+} u=\varphi_{0} \text { on } S
$$

where $\varphi_{0} \in H^{\frac{1}{2}}(S), f \in H^{0}\left(\Omega^{+}\right)$.

Neumann problem: Find a function $u \in H^{1,0}\left(\Omega^{+} ; A\right)$ satisfying the differential equation (2.6) and the boundary condition

$$
T^{+} u=\psi_{0} \text { on } S
$$

where $\psi_{0} \in H^{-\frac{1}{2}}(S)$ and $f \in H^{0}\left(\Omega^{+}\right)$.

Robin problem: Find a function $u \in H^{1,0}\left(\Omega^{+} ; A\right)$ satisfying the differential equation (2.6) and the boundary condition

$$
T^{+} u+\kappa \gamma^{+} u=\psi_{1} \text { on } S,
$$


where $\kappa$ is a smooth non-negative function which does not vanish identically on $S, \psi_{1} \in H^{-\frac{1}{2}}(S)$ and $f \in H^{0}\left(\Omega^{+}\right)$.

Equation (2.6) is understood in the distributional sense, the Dirichlet type boundary condition (2.7) is understood in the usual trace sense and the Neumann type condition in (2.8) and (2.9) for the co-normal derivative is understood in the generalized functional sense defined by relation (2.3).

Remark 2.1. It is well known that the above formulated Dirichlet and Robin BVPs are uniquely solvable, while the condition

$$
\int_{\Omega^{+}} f(x) d x=\left\langle\psi_{0}, 1\right\rangle_{S}
$$

is necessary and sufficient for the Neumann problem to be solvable (see, e.g., [27], [19], [18]). A solution of the Neumann problem is defined modulo a constant summand.

\subsection{Parametrix-based operators and auxiliary identities}

Our goal is to develop the LBDSIE method for the above formulated BVPs. To this end, let us define a localized parametrix corresponding to the fundamental solution $F(x):=-[4 \pi|x|]^{-1}$ of the Laplace operator, $\Delta=\partial_{1}^{2}+\partial_{2}^{2}+$ $\partial_{3}^{2}$,

$$
P(x) \equiv P_{\chi}(x):=\chi(x) F(x)=-\frac{\chi(x)}{4 \pi|x|} \text { with } \chi(0)=1,
$$

where $\chi$ is a localizing function which belongs to $X^{k}$ or $X_{+}^{k}$ with $k \geq 0$ (see Appendix A). Further on in the paper we will assume that $\chi \in X_{+}^{3}$ unless otherwise is explicitly stated.

Denote by $B(y, \varepsilon)$ a ball centred at a point $y$, with a radius $\varepsilon$, and let $\Sigma(y, \varepsilon):=\partial B(y, \varepsilon)$. Let us take $u \in \mathcal{D}\left(\overline{\Omega^{+}}\right)$and $v(x)=P(x-y)$, where $y$ is an arbitrarily fixed interior point in $\Omega^{+}$. For $\chi \in X^{3}$ we have (cf. Lemma B.1 and Corollary B.2) that $v \in H^{2}\left(\Omega_{\varepsilon}^{+}\right)$, where $\Omega_{\varepsilon}^{+}:=\Omega^{+} \backslash \overline{B(y, \varepsilon)}$ with $\varepsilon>0$ such that $\overline{B(y, \varepsilon)} \subset \Omega^{+}$, and thus we can write the second Green identity for the region $\Omega_{\varepsilon}^{+}$,

$$
\begin{aligned}
\int_{\Omega_{\varepsilon}^{+}}[ & \left.P(x-y) A\left(x, \partial_{x}\right) u(x)-u(x) A\left(x, \partial_{x}\right) P(x-y)\right] d x \\
= & \int_{S}\left[P(x-y) T^{+} u(x)-\left\{T\left(x, \partial_{x}\right) P(x-y)\right\} \gamma^{+} u(x)\right] d S_{x} \\
& +\int_{\Sigma(y, \varepsilon)}\left[P(x-y) T u(x)-\left\{T\left(x, \partial_{x}\right) P(x-y)\right\} u(x)\right] d S_{x} .
\end{aligned}
$$

Here the direction of the normal vector on $\Sigma(y, \varepsilon)$ is chosen outward $\Omega_{\varepsilon}^{+}$, i.e., inward $B(y, \epsilon)$. $\mathcal{N}$ as

For $\chi \in X^{3}$ and $u \in \mathcal{D}\left(\overline{\Omega^{+}}\right)$, let us define the singular integral operator

$$
\mathcal{N} u(y):=\text { v.p. } \int_{\Omega^{+}}\left[A\left(x, \partial_{x}\right) P(x-y)\right] u(x) d x
$$




$$
:=\lim _{\varepsilon \rightarrow 0} \int_{\Omega_{\varepsilon}^{+}}\left[A\left(x, \partial_{x}\right) P(x-y)\right] u(x) d x .
$$

Note that in the distributional sense,

$$
\frac{\partial^{2}}{\partial x_{k} \partial x_{j}} \frac{1}{|x-y|}=-\frac{4 \pi \delta_{k j}}{3} \delta(x-y)+\text { v.p. } \frac{\partial^{2}}{\partial x_{k} \partial x_{j}} \frac{1}{|x-y|},
$$

where $\delta_{k j}$ is the Kronecker delta, $\delta(\cdot)$ is the Dirac distribution and it is easy to check that the last term in (2.14) is well defined. Therefore, in view of (2.11) and taking into account that $\chi(0)=1$, we can write the following equality in the distributional sense

$$
\begin{aligned}
A\left(x, \partial_{x}\right) P(x-y) & =a_{k j}(x) \frac{\partial^{2} P(x-y)}{\partial x_{k} \partial x_{j}}+\frac{\partial a_{k j}(x)}{\partial x_{k}} \frac{\partial P(x-y)}{\partial x_{j}} \\
& =\beta(x) \delta(x-y)+\text { v.p. } A(x, \partial) P(x-y),
\end{aligned}
$$

where

$$
\begin{gathered}
\beta(x)=\frac{1}{3}\left[a_{11}(x)+a_{22}(x)+a_{33}(x)\right], \\
\text { v.p. } A\left(x, \partial_{x}\right) P(x-y)=\text { v.p. }\left[-\frac{a_{k j}(x)}{4 \pi} \frac{\partial^{2}}{\partial x_{k} \partial x_{j}} \frac{1}{|x-y|}\right]+R(x, y) \\
=\text { v.p. }\left[-\frac{a_{k j}(y)}{4 \pi} \frac{\partial^{2}}{\partial x_{k} \partial x_{j}} \frac{1}{|x-y|}\right]+R_{1}(x, y), \\
\begin{aligned}
R(x, y):=-\frac{1}{4 \pi}\left\{\frac{\partial}{\partial x_{k}}\left[\frac{\partial \chi(x-y)}{\partial x_{j}} \frac{a_{k j}(x)}{|x-y|}\right]\right. \\
+\frac{\partial\left[a_{k j}(x) \chi(x-y)\right]}{\partial x_{k}} \frac{\partial}{\partial x_{j}} \frac{1}{|x-y|} \\
\left.+a_{k j}(x)[\chi(x-y)-1] \frac{\partial^{2}}{\partial x_{k} \partial x_{j}} \frac{1}{|x-y|}\right\} \\
R_{1}(x, y):=R(x, y)-\frac{a_{k j}(x)-a_{k j}(y)}{4 \pi} \frac{\partial^{2}}{\partial x_{k} \partial x_{j}} \frac{1}{|x-y|} .
\end{aligned}
\end{gathered}
$$

Clearly, if $\chi \in X^{2}$, the functions $R(x, y)$ and $R_{1}(x, y)$ generally possess weak singularities of order $\mathcal{O}\left(|x-y|^{-2}\right)$ as $x \rightarrow y$. It is evident that if $a_{k j}(x)=$ $a(x) \delta_{k j}$, then the first terms in the right-hand sides of (2.16)-(2.17) vanish and v.p. $A\left(x, \partial_{x}\right) P(x-y)$ becomes a weakly singular function.

Further, by direct calculations one can easily verify that

$$
\begin{aligned}
\lim _{\varepsilon \rightarrow 0} \int_{\Sigma(y, \varepsilon)} P(x-y) T\left(x, \partial_{x}\right) u(x) d S_{x} & =0 \\
\lim _{\varepsilon \rightarrow 0} \int_{\Sigma(y, \varepsilon)}\left\{T\left(x, \partial_{x}\right) P(x-y)\right\} u(x) d S_{x} & =-\frac{u(y) a_{k j}(y)}{4 \pi} \int_{\Sigma_{1}} \eta_{k} \eta_{j} d S \\
=-\frac{u(y) a_{k j}(y)}{4 \pi} \frac{4 \pi \delta_{k j}}{3} & =-\beta(y) u(y)
\end{aligned}
$$


where $\Sigma_{1}$ is a unit sphere, $\eta=\left(\eta_{1}, \eta_{2}, \eta_{3}\right) \in \Sigma_{1}$, and $\beta$ is defined by (2.15).

Passing to the limit in (2.12) as $\varepsilon \rightarrow 0$ and using the relations (2.13), (2.18), and (2.19) we obtain the two-operator localized parametrix-based Green third identity in the form

$$
\begin{array}{r}
\beta(y) u(y)+\mathcal{N} u(y)-V\left(T^{+} u\right)(y)+W\left(\gamma^{+} u\right)(y)=\mathcal{P}(A u)(y), \\
y \in \Omega^{+}, \quad \chi \in X^{3},
\end{array}
$$

where $\mathcal{N}$ is a localized singular domain integral operator given by (2.13), while $V, W$, and $\mathcal{P}$ are the following localized single layer, double layer, and Newton-type volume potentials,

$$
\begin{aligned}
V(g)(y) & :=-\int_{S} P(x-y) g(x) d S_{x}, \\
W(g)(y) & :=-\int_{S}\left[T\left(x, \partial_{x}\right) P(x-y)\right] g(x) d S_{x}, \\
\mathcal{P}(h)(y) & :=\int_{\Omega^{+}} P(x-y) h(x) d x .
\end{aligned}
$$

If the domain of integration in the Newton-type volume potential (2.23) is the whole space $\mathbb{R}^{3}$, we employ the notation

$$
\mathbf{P}(h)(y):=\int_{\mathbb{R}^{3}} P(x-y) h(x) d x .
$$

Mapping properties of potentials (2.21)-(2.23) are investigated in [9], and we have collected in Appendix B some of them needed in our analysis.

Due to the density of $\mathcal{D}\left(\overline{\Omega^{+}}\right)$in $H^{1,0}\left(\Omega^{+} ; A\right)$ (see [24, Theorem 3.12]) and the mapping properties of the potentials, Green's third identity $(2.20)$ is valid also for $u \in H^{1,0}\left(\Omega^{+} ; A\right)$. In this case, the co-normal derivative $T^{+} u$ is understood in the sense of definition (2.3).

For $u \in H^{1}\left(\Omega^{+}\right)$and $\chi \in X^{3}$, applying the first Green identity (2.5) to definition (2.13), we derive that

$$
\mathcal{N} u(y)=-\beta(y) u(y)-W\left(\gamma^{+} u\right)(y)+\partial_{l} \mathcal{P}\left(a_{k l} \partial_{k} u\right)(y), \quad \forall y \in \Omega^{+},
$$

where we have taken into account that

$$
-\int_{\Omega^{+}} a_{k l}(x) \frac{\partial P(x-y)}{\partial x_{l}} \frac{\partial u(x)}{\partial x_{k}} d x=\partial_{l} \mathcal{P}\left(a_{k l} \partial_{k} u\right)(y), \quad \forall y \in \Omega^{+} .
$$

Keeping in mind that $\mathcal{D}(\bar{\Omega})$ is dense in $H^{s}\left(\Omega^{+}\right)$for any $s \in \mathbb{R}$, we will use relation (2.24) as a generalised definition of the operator $\mathcal{N}$ for a wider range of $s$ and for $\chi \in X^{k}$ also with $k<3$. Then the mapping properties of the operators $\gamma^{+}, \mathcal{P}$, and $W$, see Theorems B.3 and B.4, imply that the operator

$$
\mathcal{N}: H^{s}\left(\Omega^{+}\right) \rightarrow H^{s}\left(\Omega^{+}\right), \quad \frac{1}{2}<s<\frac{3}{2},
$$

is continuous if $\chi \in X^{2}$. 
For $u \in \mathcal{D}\left(\mathbb{R}^{3}\right)$ and $\chi \in X^{3}$ we can define, similar to (2.13), the singular integral operator over $\mathbb{R}^{3}$,

$$
\mathbf{N} u(y):=\text { v.p. } \int_{\mathbb{R}^{3}}\left[A\left(x, \partial_{x}\right) P(x-y)\right] u(x) d x,
$$

which by the same reasoning as for $\mathcal{N}$ can also be represented in the form

$$
\mathbf{N} u(y)=-\beta(y) u(y)+\partial_{l} \mathbf{P}\left(a_{k l} \partial_{k} u\right)(y), \quad \forall y \in \mathbb{R}^{3} .
$$

Keeping in mind that $\mathcal{D}\left(\mathbb{R}^{3}\right)$ is dense in $H^{s}\left(\mathbb{R}^{3}\right)$ for any $s \in \mathbb{R}$, we will employ (2.26) as a generalised definition of the operator $\mathbf{N}$ on $H^{s}\left(\mathbb{R}^{3}\right)$. By the mapping property (B.2) for the operator $\mathbf{P}$, we then obtain that the operator

is continuous if $\chi \in X^{1}$.

$$
\mathbf{N}: H^{s}\left(\mathbb{R}^{3}\right) \rightarrow H^{s}\left(\mathbb{R}^{3}\right), \quad s \in \mathbb{R}
$$

Denote by $E_{0}$ the extension operator by zero from $\Omega^{+}$onto $\Omega^{-}$. For a function $u \in H^{1}\left(\Omega^{+}\right)$we have

$$
\mathcal{N} u=\mathbf{N} E_{0} u
$$

where the derivatives in the right hand side of (2.27) given by definition (2.26) should be understood in the distributional sense. This (cf. also (2.25)) implies the continuity of the operator

$$
r_{\Omega^{+}} \mathbf{N} E_{0}: H^{s}\left(\Omega^{+}\right) \rightarrow H^{s}\left(\Omega^{+}\right), \quad \frac{1}{2}<s<\frac{3}{2} .
$$

Here and throughout the paper $r_{\Omega}$ denotes the restriction operator to $\Omega$. The continuity of operator (2.28) is implied also by more general assertions (see, e.g., [5], [18, Theorem 8.6.1]) due to decomposition (2.17) since the symbol of the operator $\mathbf{N}$ is rational (see (4.4)) and the operators with the kernel functions $R(x, y)$ and $R_{1}(x, y)$ map $H^{s}\left(\Omega^{+}\right) \subset H^{\frac{1}{2}-\varepsilon}\left(\Omega^{+}\right)$into $H^{\frac{3}{2}-\varepsilon}\left(\Omega^{+}\right)$ for $1 / 2<s<3 / 2$, any $\epsilon>0$, and $\chi \in X^{2}$ (cf. [9], Theorem 5.4).

Now we rewrite Green's third formula $(2.20)$ for $u \in H^{1,0}\left(\Omega^{+} ; A\right)$ in a form more convenient for our purposes:

$$
\begin{array}{r}
{[\beta \mathbf{I}+\mathbf{N}] E_{0} u(y)-V\left(T^{+} u\right)(y)+W\left(\gamma^{+} u\right)(y)=\mathcal{P}\left(A\left(x, \partial_{x}\right) u\right)(y),} \\
y \in \Omega^{+}, \chi \in X^{3},
\end{array}
$$

where I stands for the identity operator.

Using the properties of localized potentials (see Theorems B.3 and B.5) and taking the trace of equation (2.29) on $S$, we arrive for $u \in H^{1,0}\left(\Omega^{+} ; A\right)$ at the relation

$$
\begin{array}{r}
\mathbf{N}^{+} E_{0} u-\mathcal{V}\left(T^{+} u\right)+(\beta-\mu) \gamma^{+} u+\mathcal{W}\left(\gamma^{+} u\right)=\mathcal{P}^{+}\left(A\left(x, \partial_{x}\right) u\right) \\
\text { on } S, \chi \in X^{3},
\end{array}
$$

where the localized boundary integral operators $\mathcal{V}$ and $\mathcal{W}$ are generated by the single and double layer potentials,

$$
\mathcal{V} g(y):=-\int_{S} P(x-y) g(x) d S_{x}, \quad y \in S,
$$




$$
\mathcal{W} g(y):=-\int_{S}\left[T\left(x, \partial_{x}\right) P(x-y)\right] g(x) d S_{x}, \quad y \in S
$$

while

$$
\begin{aligned}
& \mu(y):=\frac{1}{2} a_{k j}(y) n_{k}(y) n_{j}(y)>0, \quad y \in S, \\
& \mathbf{N}^{+}:=\gamma^{+} \mathbf{N} \text { on } S, \quad \mathcal{P}^{+}:=\gamma^{+} \mathcal{P} \text { on } S .
\end{aligned}
$$

Now we prove the following technical lemma.

Lemma 2.2. Let $f \in H^{0}\left(\Omega^{+}\right), F \in H^{1,0}\left(\Omega^{+} ; \Delta\right), \psi \in H^{-\frac{1}{2}}(S), \varphi \in H^{\frac{1}{2}}(S)$, and $\chi \in X^{3}$. Moreover, let $u \in H^{1}\left(\Omega^{+}\right)$and the following equation hold

$$
\begin{array}{r}
\beta(y) u(y)+\mathcal{N} u(y)-V(\psi)(y)+W(\varphi)(y)=F(y)+\mathcal{P}(f)(y), \\
y \in \Omega^{+} .
\end{array}
$$

Then $u \in H^{1,0}\left(\Omega^{+} ; A\right)$ and the following estimate holds

$$
\begin{aligned}
\|u\|_{H^{1,0}\left(\Omega^{+} ; A\right)} & :=\|u\|_{H^{1}\left(\Omega^{+}\right)}+\|A u\|_{H^{0}\left(\Omega^{+}\right)} \\
\leq C\left(\|u\|_{H^{1}\left(\Omega^{+}\right)}+\|\psi\|_{H^{-\frac{1}{2}}(\partial \Omega)}+\|\varphi\|_{H^{\frac{1}{2}}(\partial \Omega)}\right. & \left.+\|f\|_{H^{0}\left(\Omega^{+}\right)}+\|F\|_{H^{1,0}\left(\Omega^{+} ; \Delta\right)}\right) \\
& +{ }^{\leq}
\end{aligned}
$$

with some positive constant $C$.

Proof. In view of definition (2.24), equation (2.34) can be rewritten as

$$
\begin{array}{r}
\partial_{l} \mathcal{P}\left(a_{k l} \partial_{k} u\right)(y)=F(y)+\mathcal{P}(f)(y)+V(\psi)(y)-W\left(\varphi-\gamma^{+} u\right)(y), \\
y \in \Omega^{+} .
\end{array}
$$

By Theorem B.3, $\mathcal{P}(f) \in H^{2}\left(\Omega^{+}\right)$for arbitrary $f \in H^{0}\left(\Omega^{+}\right)$, while the inclusions $V(\psi), W(\varphi) \in H^{1,0}\left(\Omega^{+} ; \Delta\right)$ hold for arbitrary $\psi \in H^{-\frac{1}{2}}(S)$ and $\varphi \in H^{\frac{1}{2}}(S)$ by Theorem B.4 . This implies that the right-hand side function in (2.36) belongs to the space $H^{1,0}\left(\Omega^{+} ; \Delta\right)$ since $\gamma^{+} u \in H^{\frac{1}{2}}(S)$, and therefore

$$
\partial_{l} \mathcal{P}\left(a_{k l} \partial_{k} u\right) \in H^{1,0}\left(\Omega^{+} ; \Delta\right) \text {. }
$$

We have

$$
\Delta_{x} P(x-y)=\Delta_{y} P(x-y)=\delta(x-y)+R_{\Delta}(x-y),
$$

where

$$
R_{\Delta}(x-y):=-\frac{1}{4 \pi}\left\{\frac{\Delta_{x} \chi(x-y)}{|x-y|}+2 \frac{\partial \chi(x-y)}{\partial x_{l}} \frac{\partial}{\partial x_{l}} \frac{1}{|x-y|}\right\} .
$$

Clearly, $R_{\Delta}(x-y)=\mathcal{O}\left(|x-y|^{-2}\right)$ as $x \rightarrow y$ and with the help of (2.38) and (2.39) one can establish that for arbitrary $\Phi \in \mathcal{D}\left(\Omega^{+}\right)$there holds the relation (see, e.g., [26])

$$
(\Delta \mathcal{P} \Phi)(y)=\Phi(y)+\left(\mathcal{R}_{\Delta} \Phi\right)(y), \quad y \in \Omega^{+},
$$

where

$$
\left(\mathcal{R}_{\Delta} \Phi\right)(y):=\int_{\Omega^{+}} R_{\Delta}(x-y) \Phi(x) d x
$$


Evidently (2.40) remains true also for $\Phi \in H^{0}\left(\Omega^{+}\right)$, since $\mathcal{D}\left(\Omega^{+}\right)$is dense in $H^{0}\left(\Omega^{+}\right)$and the operators

$$
\mathcal{R}_{\Delta}: H^{0}\left(\Omega^{+}\right) \rightarrow H^{1}\left(\Omega^{+}\right), \quad \mathcal{P}: H^{0}\left(\Omega^{+}\right) \rightarrow H^{2}\left(\Omega^{+}\right),
$$

are bounded if $\chi \in X^{2}$ (cf. [9, Theorem 5.4]). Further,

$$
\begin{aligned}
\Delta\left[\partial_{l} \mathcal{P}\left(a_{k l} \partial_{k} u\right)\right. & (y)]=\partial_{l}\left[\Delta \mathcal{P}\left(a_{k l} \partial_{k} u\right)(y)\right] \\
= & \partial_{l}\left[a_{k l}(y) \partial_{k} u(y)\right]+\partial_{l} \mathcal{R}_{\Delta}\left(a_{k l} \partial_{k} u\right)(y) \\
= & A\left(y, \partial_{y}\right) u(y)+\partial_{l} \mathcal{R}_{\Delta}\left(a_{k l} \partial_{k} u\right)(y), \quad y \in \Omega^{+} .
\end{aligned}
$$

Whence the embedding $A\left(y, \partial_{y}\right) u \in H^{0}\left(\Omega^{+}\right)$and estimate (2.35) follow due to $(2.36),(2.37),(2.41)$ and Theorem B.4.

In fact, the continuity of the first operator in (2.41) and identity (2.42) in the proof of Lemma 2.2 imply by definition (2.24) the following assertion.

Corollary 2.3. If $\chi \in X^{3}$, then the following operator is bounded

$$
\beta \mathbf{I}+\mathcal{N}: H^{1,0}\left(\Omega^{+} ; A\right) \rightarrow H^{1,0}\left(\Omega^{+} ; \Delta\right) .
$$

\section{BVPs reduction to LBDSIE systems and equivalence theorems}

Now, we are in the position to reduce equivalently the boundary value problems to the LBDSIE systems.

\subsection{LBDSIE system for the Dirichlet problem and the equivalence theorem}

Let $u \in H^{1,0}\left(\Omega^{+} ; A\right)$ be a solution to the Dirichlet BVP (2.6)-(2.7) with $\varphi_{0} \in H^{\frac{1}{2}}(S)$ and $f \in H^{0}\left(\Omega^{+}\right)$. Relations (2.29) and (2.30) can be rewritten in the form

$$
\begin{aligned}
& {[\beta \mathbf{I}+\mathbf{N}] E_{0} u-V(\psi)=\mathcal{P}(f)-W\left(\varphi_{0}\right) \text { in } \Omega^{+},} \\
& \mathbf{N}^{+} E_{0} u-\mathcal{V}(\psi)=\mathcal{P}^{+}(f)-(\beta-\mu) \varphi_{0}-\mathcal{W}\left(\varphi_{0}\right) \text { on } S,
\end{aligned}
$$

where $\psi:=T^{+} u \in H^{-\frac{1}{2}}(S)$ and $\mu$ is defined by (2.33). One can consider these relations as a LBDSIE system with respect to the segregated unknown functions $u$ and $\psi$.

Theorem 3.1. Let $\chi \in X_{+}^{3}, \varphi_{0} \in H^{\frac{1}{2}}(S)$, and $f \in H^{0}\left(\Omega^{+}\right)$.

(i) If a function $u \in H^{1,0}\left(\Omega^{+} ; A\right)$ solves the Dirichlet $B V P(2.6)-(2.7)$, then the solution is unique and the pair $(u, \psi) \in H^{1,0}\left(\Omega^{+} ; A\right) \times H^{-\frac{1}{2}}(S)$ with

$$
\psi=T^{+} u
$$

solves LBDSIE system (3.1)-(3.2).

(ii) Vice versa, if a pair $(u, \psi) \in H^{1,0}\left(\Omega^{+} ; A\right) \times H^{-\frac{1}{2}}(S)$ solves LBDSIE system (3.1)-(3.2), then the solution is unique and the function $u$ solves the Dirichlet BVP (2.6)-(2.7), while equation (3.3) holds. 
Proof. (i) The first part of the theorem directly follows from the relations (2.29), (2.30), and (3.3).

(ii) Now, let a pair $(u, \psi) \in H^{1,0}\left(\Omega^{+} ; A\right) \times H^{-\frac{1}{2}}(S)$ solve LBDSIE system (3.1)-(3.2). Taking the trace of (3.1) on $S$ and comparing with (3.2) we get

$$
\gamma^{+} u=\varphi_{0} \text { on } S \text {. }
$$

Further, since $u \in H^{1,0}\left(\Omega^{+} ; A\right)$, we can write Green's third formula (2.29) which in view of $(3.4)$ can be rewritten as

$$
[\beta \mathbf{I}+\mathbf{N}] E_{0} u-V\left(T^{+} u\right)=\mathcal{P}\left(A\left(x, \partial_{x}\right) u\right)-W\left(\varphi_{0}\right) \text { in } \Omega^{+} .
$$

From (3.1) and (3.5) it follows that

$$
V\left(T^{+} u-\psi\right)+\mathcal{P}\left(A\left(x, \partial_{x}\right) u-f\right)=0 \text { in } \Omega^{+} .
$$

Whence by Lemma B.6 we have

$$
A\left(x, \partial_{x}\right) u=f \text { in } \Omega^{+} \text {and } T^{+} u=\psi \text { on } S .
$$

Thus $u$ solves the Dirichlet BVP (2.6)-(2.7) and equation (3.3) holds.

The uniqueness of solution to LBDSIE system (3.1)-(3.2) in the class $H^{1,0}\left(\Omega^{+} ; A\right) \times H^{-\frac{1}{2}}(S)$ directly follows from the above proved equivalence result and the uniqueness theorem for the Dirichlet problem (2.6)-(2.7) (see Remark 2.1).

\subsection{LBDSIE system for the Neumann problem and the equivalence theorem} Let $u \in H^{1,0}\left(\Omega^{+} ; A\right)$ be a solution to the Neumann BVP (2.6), (2.8) with $\psi_{0} \in H^{-\frac{1}{2}}(S)$ and $f \in H^{0}\left(\Omega^{+}\right)$. The relations (2.29) and (2.30) can be rewritten then in the form

$$
\begin{aligned}
& {[\beta \mathbf{I}+\mathbf{N}] E_{0} u+W(\varphi)=\mathcal{P}(f)+V\left(\psi_{0}\right) \text { in } \Omega^{+},} \\
& \mathbf{N}^{+} E_{0} u+(\beta-\mu) \varphi+\mathcal{W}(\varphi)=\mathcal{P}^{+}(f)+\mathcal{V}\left(\psi_{0}\right) \text { on } S,
\end{aligned}
$$

where $\varphi:=\gamma^{+} u \in H^{\frac{1}{2}}(S)$. One can consider these relations as a LBDSIE system with respect to the unknown functions $u$ and $\varphi$.

Theorem 3.2. Let $\chi \in X_{+}^{3}, \psi_{0} \in H^{-\frac{1}{2}}(S)$, and $f \in H^{0}\left(\Omega^{+}\right)$.

(i) If a function $u \in H^{1,0}\left(\Omega^{+} ; A\right)$ solves the Neumann $B V P(2.6),(2.8)$, then the pair $(u, \varphi) \in H^{1,0}\left(\Omega^{+} ; A\right) \times H^{\frac{1}{2}}(S)$ with

$$
\varphi=\gamma^{+} u \text {, }
$$

solves LBDSIE system (3.6)-(3.7).

(ii) Vice versa, if a pair $(u, \varphi) \in H^{1,0}\left(\Omega^{+} ; A\right) \times H^{\frac{1}{2}}(S)$ solves LBDSIE system (3.6)-(3.7), then the function $u$ solves the Neumann BVP (2.6), (2.8) and equality (3.8) holds.

(iii) The corresponding homogeneous LBDSIE system,

$$
\begin{aligned}
& {[\beta \mathbf{I}+\mathbf{N}] E_{0} u+W(\varphi)=0 \text { in } \Omega^{+},} \\
& \mathbf{N}^{+} E_{0} u+(\beta-\mu) \varphi+\mathcal{W}(\varphi)=0 \text { on } S,
\end{aligned}
$$


admits only one non-trivial linearly independent solution $(u, \varphi)=(1,1)$ in $H^{1,0}\left(\Omega^{+} ; A\right) \times H^{\frac{1}{2}}(S)$.

(iv) Condition (2.10) is necessary and sufficient for solvability of the nonhomogeneous LBDSIE system (3.6)-(3.7) in $H^{1,0}\left(\Omega^{+} ; A\right) \times H^{\frac{1}{2}}(S)$.

Proof. (i) The first part of the theorem directly follows from relations (2.29), (2.30), and (3.8).

(ii) Now let a pair $(u, \varphi) \in H^{1,0}\left(\Omega^{+} ; A\right) \times H^{\frac{1}{2}}(S)$ solve LBDSIE system (3.6)-(3.7). Take the trace of (3.6) on $S$ and compare with (3.7) to obtain (3.8). Further, since $u \in H^{1,0}\left(\Omega^{+} ; A\right)$, we can write Green's third identity (2.29) which in view of (3.8) takes form

$$
[\beta \mathbf{I}+\mathbf{N}] E_{0} u-V\left(T^{+} u\right)+W(\varphi)=\mathcal{P}\left(A\left(x, \partial_{x}\right) u\right) \text { in } \Omega^{+} .
$$

From (3.6) and (3.11) we get

$$
V\left(T^{+} u-\psi_{0}\right)+\mathcal{P}\left(A\left(x, \partial_{x}\right) u-f\right)=0 \text { in } \Omega^{+},
$$

and by Lemma B.6

$$
A\left(x, \partial_{x}\right) u=f \text { in } \Omega^{+} \text {and } T^{+} u=\psi_{0} \text { on } S .
$$

Thus, $u$ solves the Neumann BVP (2.6), (2.8) and equality (3.8) holds.

(iii) Let $(u, \varphi) \in H^{1,0}\left(\Omega^{+} ; A\right) \times H^{\frac{1}{2}}(S)$ solve the homogeneous LBDSIE system (3.9)-(3.10). Evidently the zero right hand sides of the system can be considered as generated by the zero right hand side, $\left(\psi_{0}, f\right)=(0,0)$, of the homogeneous Neumann BVP (2.6), (2.8). Then the already proved statements of item (ii) imply that $u$ is a solution of the homogeneous Neumann problem, which is a constant by Remark 2.1 , and thus the couple $(u, \varphi)$ is proportional to $(1,1)$ due to $(3.8)$.

(iv) By Remark 2.1, condition (2.10) implies solvability of the Neumann problem and by item (i) solvability of LBDSIE system (3.6)-(3.7) follows. Conversely, by item (ii), solvability of LBDSIE system (3.6)-(3.7) implies solvability of the Neumann problem and therefore the condition (2.10) follows due to Remark 2.1 .

\subsection{LBDSIE system for the Robin problem and the equivalence theorem}

Let $u \in H^{1,0}\left(\Omega^{+} ; A\right)$ be a solution to the Robin BVP $(2.6),(2.9)$ with $\psi_{1} \in$ $H^{-\frac{1}{2}}(S), f \in H^{0}\left(\Omega^{+}\right)$, and $\kappa$ being a smooth nonnegative nonzero function on $S$. The relations (2.29) and (2.30) can be rewritten as

$$
\begin{aligned}
& {[\beta \mathbf{I}+\mathbf{N}] E_{0} u+W(\varphi)+V(\kappa \varphi)=\mathcal{P}(f)+V\left(\psi_{1}\right) \text { in } \Omega^{+},} \\
& \mathbf{N}^{+} E_{0} u+(\beta-\mu) \varphi+\mathcal{W}(\varphi)+\mathcal{V}(\kappa \varphi)=\mathcal{P}^{+}(f)+\mathcal{V}\left(\psi_{1}\right) \text { on } S,
\end{aligned}
$$

where $\varphi:=\gamma^{+} u \in H^{\frac{1}{2}}(S)$ and we have taken into account that $T^{+} u=$ $\psi_{1}-\kappa \varphi \in H^{-\frac{1}{2}}(S)$ in accordance with the boundary condition (2.9). One can consider these relations as the LBDSIE system with respect to the unknown functions $u$ and $\varphi$. 
Theorem 3.3. Let $\chi \in X_{+}^{3}, \psi_{1} \in H^{-\frac{1}{2}}(S)$, and $f \in H^{0}\left(\Omega^{+}\right)$.

(i) If a function $u \in H^{1,0}\left(\Omega^{+} ; A\right)$ solves the Robin BVP (2.6), (2.9), then the solution is unique and the pair $(u, \varphi) \in H^{1,0}\left(\Omega^{+} ; A\right) \times H^{\frac{1}{2}}(S)$ with

$$
\varphi=\gamma^{+} u
$$

solves LBDSIE system (3.12)-(3.13).

(ii) Vice versa, if a pair $(u, \varphi) \in H^{1,0}\left(\Omega^{+} ; A\right) \times H^{\frac{1}{2}}(S)$ solves LBDSIE system (3.12)-(3.13), then the solution is unique and the function $u$ solves the Robin BVP (2.6), (2.9) and equality (3.14) holds.

Proof. (i) The first part of the theorem directly follows from relations (2.29), (2.30), and (3.14).

(ii) Now, let a pair $(u, \varphi) \in H^{1,0}\left(\Omega^{+} ; A\right) \times H^{\frac{1}{2}}(S)$ solve LBDSIE system (3.12)-(3.13). Taking the trace of (3.12) on $S$ and comparing with (3.13) lead to equation (3.14). Further, since $u \in H^{1,0}\left(\Omega^{+} ; A\right)$ we can write Green's third identity (2.29),

$$
[\beta \mathbf{I}+\mathbf{N}] E_{0} u-V\left(T^{+} u\right)+W(\varphi)=\mathcal{P}\left(A\left(x, \partial_{x}\right) u\right) \text { in } \Omega^{+},
$$

where the equality (3.14) is taken into consideration. From (3.12) and (3.15) now it follows that

$$
V\left(T^{+} u+\kappa \varphi-\psi_{1}\right)+\mathcal{P}\left(A\left(x, \partial_{x}\right) u-f\right)=0 \text { in } \Omega^{+},
$$

whence by Lemma B.6 we infer

$$
A\left(x, \partial_{x}\right) u=f \text { in } \Omega^{+} \text {and } T^{+} u+\kappa \varphi=\psi_{1} \text { on } S .
$$

Due to (3.14) we see that $u$ solves the Robin BVP (2.6), (2.9).

The uniqueness of solution for LBDSIE system (3.12)-(3.13) in the class $H^{1,0}\left(\Omega^{+} ; A\right) \times H^{\frac{1}{2}}(S)$ directly follows from the above proved equivalence result and the uniqueness theorem for the Robin BVP (2.6), (2.9) (see Remark $2.1)$.

\section{Symbols and Fredholm properties of the domain operators}

Let $\mathcal{F}$ and $\mathcal{F}^{-1}$ denote the distributional direct and inverse Fourier transform operators, that for $g \in L_{1}\left(\mathbb{R}^{3}\right)$ and $\hat{g} \in L_{1}\left(\mathbb{R}^{3}\right)$ take form

$$
[\mathcal{F} g](\xi)=\int_{\mathbb{R}^{3}} g(z) e^{i z \cdot \xi} d z, \quad\left[\mathcal{F}^{-1} \hat{g}\right](z)=\frac{1}{(2 \pi)^{3}} \int_{\mathbb{R}^{3}} \hat{g}(z) e^{-i z \cdot \xi} d \xi
$$

The complete symbol $\mathfrak{S}(\mathbf{P})$ of the operator $\mathbf{P}$ reads as, cf. [9],

$$
\begin{aligned}
\mathfrak{S}(\mathbf{P})(\xi) \equiv \mathfrak{S}(\mathbf{P} ; \xi) & :=\mathcal{F}_{x \rightarrow \xi}\left[-\frac{1}{4 \pi} \frac{\chi(x)}{|x|}\right] \\
& =-\frac{1}{4 \pi} \int_{\mathbb{R}^{3}} \frac{\chi(x)}{|x|} e^{i x \cdot \xi} d x, \quad \xi \in \mathbb{R}^{3},
\end{aligned}
$$

while its principal homogeneous symbol is (see, e.g., [15])

$$
\mathfrak{S}_{0}(\mathbf{P} ; \xi)=\mathfrak{S}\left(\mathbf{P}_{\Delta} ; \xi\right):=\mathcal{F}_{x \rightarrow \xi}\left[-\frac{1}{4 \pi|x|}\right]=-\frac{1}{|\xi|^{2}}, \quad \xi \in \mathbb{R}^{3},
$$


where $\mathbf{P}_{\Delta}$ is the classical Newton potential operator,

$$
\mathbf{P}_{\Delta} h(y):=-\frac{1}{4 \pi} \int_{\mathbb{R}^{3}} \frac{1}{|x-y|} \psi(x) d x .
$$

In what follows, we need an explicit expression of the principal homogeneous symbol $\mathfrak{S}_{0}(\mathcal{N})(y, \xi)$ of the singular integral operator $\mathcal{N}$, which due to $(2.13)-(2.17)$ and $(4.2)$ reads as

$$
\begin{aligned}
\mathfrak{S}_{0}(\mathcal{N})(y, \xi) & =\mathfrak{S}_{0}(\mathbf{N})(y, \xi)=\mathcal{F}_{z \rightarrow \xi}\left[-\frac{a_{k l}(y)}{4 \pi} \mathrm{v} \cdot \mathrm{p} \cdot \frac{\partial^{2}}{\partial z_{k} \partial z_{l}} \frac{1}{|z|}\right] \\
& =-\frac{a_{k l}(y)}{4 \pi} \mathcal{F}_{z \rightarrow \xi}\left[\frac{4 \pi \delta_{k l}}{3} \delta(z)+\frac{\partial^{2}}{\partial z_{k} \partial z_{l}} \frac{1}{|z|}\right] \\
& =-\beta(y)+a_{k l}(y) \xi_{k} \xi_{l} \mathcal{F}_{z \rightarrow \xi}\left[\frac{1}{4 \pi|z|}\right] \\
& =-\beta(y)+\frac{a_{k l}(y) \xi_{k} \xi_{l}}{|\xi|^{2}} \\
& =\frac{A(y, \xi)}{|\xi|^{2}}-\beta(y), y \in \Omega^{+}, \quad \xi \in \mathbb{R}^{3} \backslash\{0\},
\end{aligned}
$$

where we also have taken into account that $\mathcal{F}_{z \rightarrow \xi}[\delta(z)]=1$.

As we see, the principal homogeneous symbol $\mathfrak{S}_{0}(\mathcal{N})(y, \xi)$ of the operator $\mathcal{N}$ is an even rational homogeneous function in $\xi$ of order 0 . It can easily be verified that both the characteristic function of the singular kernel in (4.3) and the Fourier transform (4.4) satisfy the Tricomi condition, i.e., their integral averages over the unit sphere vanish (cf. [26]).

Relation (4.4) implies that the principal homogeneous symbols of the singular integral operators $\mathbf{N}$ and $\beta \mathbf{I}+\mathbf{N}$ read as

$$
\begin{array}{lll}
\mathfrak{S}_{0}(\mathbf{N})(y, \xi)=\frac{A(y, \xi)-\beta|\xi|^{2}}{|\xi|^{2}} & \forall y \in \mathbb{R}^{3}, \quad \forall \xi \in \mathbb{R}^{3} \backslash\{0\}, \\
\mathfrak{S}_{0}(\beta \mathbf{I}+\mathbf{N})(y, \xi)=\frac{A(y, \xi)}{|\xi|^{2}}>0 & \forall y \in \mathbb{R}^{3}, \quad \forall \xi \in \mathbb{R}^{3} \backslash\{0\} .
\end{array}
$$

Let us introduce the notation $\mathcal{B}:=r_{\Omega^{+}}(\beta \mathbf{I}+\mathbf{N}) E_{0}$.

Lemma 4.1. Let a localizing function $\chi \in X^{\infty}$. The operator

$$
\mathcal{B}: H^{s}\left(\Omega^{+}\right) \rightarrow H^{s}\left(\Omega^{+}\right)
$$

is Fredholm with zero index for all $s \geq 0$.

Proof. The principal homogeneous symbol of the operator $\mathcal{B}$ (cf. (4.6)) can be written as

$$
\begin{array}{r}
\mathfrak{S}_{0}(\mathcal{B})(y, \xi)=\mathfrak{S}_{0}(\beta \mathbf{I}+\mathbf{N})(y, \xi)=\frac{A(y, \xi)}{|\xi|^{2}}=\frac{a_{k l}(y) \xi_{k} \xi_{l}}{\xi_{1}^{2}+\xi_{2}^{2}+\xi_{3}^{2}}>0, \\
y \in \overline{\Omega^{+}}, \quad \xi \in \mathbb{R}^{3} \backslash\{0\} .
\end{array}
$$


We see that the symbol (4.8) is an even rational homogeneous function of order 0 in $\xi$. Then the factorization index $\varkappa$ of the symbol (4.8) equals to zero (see [15], $\S 6$ ). Moreover, since (4.8) is rational function in $\xi$, the operator $\mathcal{B}$ possesses the transmission property. Therefore we can apply the theory of pseudodifferential operators satisfying the transmission property (see [15], Theorem 11.1, and Lemma 23.9; [5], [28]) to deduce that the operator (4.7) is Fredholm for all $s \geq 0$.

To show that Ind $\mathcal{B}=0$ we use the fact that the operators $\mathcal{B}$ and

$$
\mathcal{B}_{t}=r_{\Omega^{+}}[(1-t) \mathbf{I}+t(\beta \mathbf{I}+\mathbf{N})] E_{0}, \quad t \in[0,1],
$$

are homotopic in the sense of the definition from $\left[26\right.$, p. 27]. Note that $\mathcal{B}_{0}=$ $r_{\Omega^{+}} \mathbf{I} E_{0}$ and $\mathcal{B}_{1}=\mathcal{B}$.

In view of (4.5) and (4.6) it is easy to see that

$$
\mathfrak{S}_{0}\left(\mathcal{B}_{t}\right)(y, \xi)=\frac{(1-t)|\xi|^{2}+t A(y, \xi)}{|\xi|^{2}}>0
$$

i.e. the operator $\mathcal{B}_{t}$ is elliptic, for all $t \in[0,1]$, for all $y \in \overline{\Omega^{+}}$, and for all $\xi \in \mathbb{R}^{3} \backslash\{0\}$.

Since $\mathfrak{S}_{0}\left(\mathcal{B}_{t}\right)(y, \xi)$ is rational, even, and homogeneous of order zero in $\xi$, we conclude that the operator $\mathcal{B}_{t}: H^{s}\left(\Omega^{+}\right) \rightarrow H^{s}\left(\Omega^{+}\right)$is Fredholm for all $s \geq 0$ and for all $t \in[0,1]$. Therefore Ind $\mathcal{B}_{t}$ is the same for all $t \in[0,1]$ (see e.g. [26, Chapter 1, Theorem 3.11]). On the other hand, due to the equality $\mathcal{B}_{0}=\mathbf{I}$ we get Ind $\mathcal{B}=\operatorname{Ind} \mathcal{B}_{1}=\operatorname{Ind} \mathcal{B}_{t}=\operatorname{Ind} \mathcal{B}_{0}=0$.

\section{Invertibility of the LBDSIO for the Dirichlet problem}

From Theorem 3.1 it follows that the LBDSIE system (3.1)-(3.2), which has a special right-hand side, is uniquely solvable in the class $H^{1,0}\left(\Omega^{+} ; A\right) \times$ $H^{-\frac{1}{2}}(S)$. However, this fact does not imply invertibility of the LBDSIO generated by the left-hand side expressions in (3.1)-(3.2). In what follows, we investigate Fredholm properties of this operator and establish invertibility results in appropriate function spaces. To this end, we consider the LBDSIE system

$$
\begin{aligned}
& {[\beta \mathbf{I}+\mathbf{N}] E_{0} u-V(\psi)=F_{1} \text { in } \Omega^{+},} \\
& \mathbf{N}^{+} E_{0} u-\mathcal{V}(\psi)=F_{2} \text { on } S,
\end{aligned}
$$

where $F_{1} \in H^{1}\left(\Omega^{+}\right)$and $F_{2} \in H^{\frac{1}{2}}(S)$ are arbitrary functions.

Denote by $\mathfrak{D}$ the localized boundary-domain integral operator generated by the left-hand side expressions in LBDSIE system (5.1)-(5.2),

$$
\mathfrak{D}:=\left[\begin{array}{ll}
r_{\Omega^{+}}(\beta \mathbf{I}+\mathbf{N}) E_{0} & -r_{\Omega^{+}} V \\
\mathbf{N}^{+} E_{0} & -\mathcal{V}
\end{array}\right]
$$

and consider the operator

$$
\mathfrak{D}: H^{r+1}\left(\Omega^{+}\right) \times H^{r-\frac{1}{2}}(S) \rightarrow H^{r+1}\left(\Omega^{+}\right) \times H^{r+\frac{1}{2}}(S) .
$$


First of all note that if a localizing function is infinitely differentiable in the radial direction, $\chi \in X^{\infty}$, then the localized volume and layer potentials and the corresponding boundary operators have exactly the same mapping properties as the corresponding harmonic potentials (see, e.g., [5], [6, Theorem 5.2.4(i)], [18, Theorem 8.4.13, 8.6.1], [27, Ch. 2]). Therefore the operator (5.3) is continuous for all $r>-\frac{1}{2}$ if $\chi \in X^{\infty}$.

In what follows, we employ the approach considered in $\S 12, \S 22$, and $\S 23$ of the monograph [15] and prove the invertibility of operator (5.3) for $r>-1 / 2$ and $\chi \in X_{+}^{\infty}$ in three steps. First we prove that the operator is Fredholm, then show that its index is zero and that the operator is injective. Then we prove the invertibility for $r=0$ when $\chi \in X_{+}^{3}$.

Let us introduce two auxiliary operators. The operator $\Pi^{+}$is a Cauchy type integral operator

$$
\Pi^{+}(h)(\xi):=\frac{i}{2 \pi} \lim _{t \rightarrow 0+} \int_{-\infty}^{+\infty} \frac{h\left(\xi^{\prime}, \eta_{3}\right) d \eta_{3}}{\xi_{3}+i t-\eta_{3}}, \quad \xi^{\prime}:=\left(\xi_{1}, \xi_{2}\right) \in \mathbb{R}^{3},
$$

which is well defined for a bounded smooth function $h\left(\xi^{\prime}, \cdot\right)$ satisfying the relation $h\left(\xi^{\prime}, \eta_{3}\right)=\mathcal{O}\left(\left(1+\left|\eta_{3}\right|\right)^{-\nu}\right)$ with some $\nu>0$.

The operator $\Pi^{\prime}$ is defined as

$$
\begin{aligned}
\Pi^{\prime}(g)\left(\xi^{\prime}\right) & :=\lim _{x_{3} \rightarrow 0+} r_{\mathbb{R}_{+}^{3}} \mathcal{F}_{\xi_{3} \rightarrow x_{3}}^{-1}\left[g\left(\xi^{\prime}, \xi_{3}\right)\right] \\
& =\frac{1}{2 \pi} \lim _{x_{3} \rightarrow 0+} \int_{-\infty}^{+\infty} g\left(\xi^{\prime}, \xi_{3}\right) e^{-i x_{3} \xi_{3}} d \xi_{3} \\
& =\frac{1}{2 \pi} \int_{-\infty}^{+\infty} g\left(\xi^{\prime}, \xi_{3}\right) d \xi_{3} \text { for } g\left(\xi^{\prime}, \cdot\right) \in L_{1}(\mathbb{R})
\end{aligned}
$$

The operator $\Pi^{\prime}$ can be extended to the class of functions $g\left(\xi^{\prime}, \xi_{3}\right)$ being rational in $\xi_{3}$ with the denominator not vanishing for real non-zero $\xi=$ $\left(\xi^{\prime}, \xi_{3}\right) \in \mathbb{R}^{3} \backslash\{0\}$, homogeneous of order $m \in \mathbb{Z}:=\{0, \pm 1, \pm 2, \ldots\}$ in $\xi$, and infinitely differentiable with respect to $\xi$ for $\xi^{\prime} \neq 0$. Then one can show that (see Appendix C for details)

$$
\Pi^{\prime}(g)\left(\xi^{\prime}\right)=\lim _{x_{3} \rightarrow 0+} r_{\mathbb{R}_{+}} \mathcal{F}_{\xi_{3} \rightarrow x_{3}}^{-1}\left[g\left(\xi^{\prime}, \xi_{3}\right)\right]=-\frac{1}{2 \pi} \int_{\Gamma^{-}} g\left(\xi^{\prime}, \zeta\right) d \zeta,
$$

where $r_{\mathbb{R}_{+}}$denotes the restriction operator onto $\mathbb{R}_{+}=(0,+\infty)$ with respect to $x_{3}, \Gamma^{-}$is a contour in the lower complex half-plane in $\zeta$, orientated anticlockwise and enclosing all the poles of the rational function $g\left(\xi^{\prime}, \cdot\right)$. It is clear that if $g\left(\xi^{\prime}, \zeta\right)$ is holomorphic in $\zeta$ in the lower complex half-plane $(\operatorname{Im} \zeta<0)$, then $\Pi^{\prime}(g)\left(\xi^{\prime}\right)=0$.

Now we prove the following basic assertion (cf. [15], Theorem 23.1).

Lemma 5.1. Let a localizing function $\chi \in X^{\infty}$ and $r>-\frac{1}{2}$. Then operator (5.3) is Fredholm with zero index.

Proof. To investigate Fredholm properties of the operator $\mathfrak{D}$, we will employ the results of $\S 12, \S 22$, and $\S 23$ in [15], based on the local principle. 
In particular, we will essentially apply Theorems 12.2 and 23.1 in [15] along with formulas (12.25) and (12.27) from the same book (note that $\stackrel{\circ}{H}_{r}(G)$ should evidently read as $H_{r}(G)$ in Theorem 23.1, as in the original Russian edition of the book [15]).

Since the operator $\mathcal{B}=r_{\Omega^{+}}(\beta \mathbf{I}+\mathbf{N}) E_{0}$ is Fredholm with zero index due to Lemma 4.1 and its principal homogeneous symbol is rational, from the above mentioned theorems in [15] it follows that operator (5.3) is Fredholm for $r>-1 / 2$ if in a local coordinate system with the origin at an arbitrary "frozen" point $\widetilde{y} \in S=\partial \Omega$ the following generalized Šapiro-Lopatinskii condition holds,

$$
\begin{aligned}
e\left(\widetilde{y}, \xi^{\prime}\right)= & -\Pi^{\prime}\left[\frac{\mathfrak{S}_{0}(\widetilde{\mathbf{N}})(\widetilde{y}, \cdot)}{\mathfrak{S}_{0}^{(+)}(\widetilde{\mathcal{B}})(\widetilde{y}, \cdot)} \Pi^{+}\left(\frac{\mathfrak{S}_{0}(\widetilde{\mathbf{P}})(\widetilde{y}, \cdot)}{\mathfrak{S}_{0}^{(-)}(\widetilde{\mathcal{B}})(\widetilde{y}, \cdot)}\right)\right]\left(\xi^{\prime}\right) \\
& -\mathfrak{S}_{0}(\widetilde{\mathcal{V}})\left(\widetilde{y}, \xi^{\prime}\right) \neq 0, \quad \forall \xi^{\prime} \neq 0, \quad \forall \widetilde{y} \in \partial \Omega,
\end{aligned}
$$

where $\widetilde{\mathbf{N}}, \widetilde{\mathcal{B}}, \widetilde{\mathbf{P}}$, and $\widetilde{\mathcal{V}}$ denote the operators $\mathbf{N}, \mathcal{B}, \mathbf{P}$, and $\mathcal{V}$ respectively, written in the chosen local coordinate system, while $\mathfrak{S}_{0}(\widetilde{\mathbf{N}}), \mathfrak{S}_{0}(\widetilde{\mathcal{B}}), \mathfrak{S}_{0}(\widetilde{\mathbf{P}})$, and $\mathfrak{S}_{0}(\widetilde{\mathcal{V}})$ are the corresponding principal homogeneous symbols in the same local coordinate system; $\mathfrak{S}_{0}^{( \pm)}(\widetilde{\mathcal{B}})$ denote the so called "plus" and "minus" factors in the factorization of the symbol $\mathfrak{S}_{0}(\widetilde{\mathcal{B}})$ with respect to the variable $\xi_{3}$. Here we also have taken into account the representations (B.6) and (B.7) for the single layer potential.

We assume that these symbols are "frozen" at the point $\widetilde{y} \in S$ considered as the origin $\widetilde{O}$ of the local coordinate system. If the matrix of transformation of the original coordinate system $O y_{1} y_{2} y_{3}$ to the new one $\widetilde{O} \eta_{1} \eta_{2} \eta_{3}$ is an orthogonal matrix $\Lambda(\widetilde{y}):=\left[\lambda_{k l}(\widetilde{y})\right]_{3 \times 3}$, which transforms the outward unit normal vector $n^{\top}(\widetilde{y})$ into the vector $\mathbf{e}_{3}=(0,0,-1)^{\top}$ (the outward unit normal vector to $\left.\mathbb{R}_{+}^{3}\right)$, i.e., $n^{\top}(\widetilde{y})=\Lambda(\widetilde{y}) \mathbf{e}_{3}$, then

$$
\begin{aligned}
& \lambda_{k 3}(\widetilde{y})=-n_{k}(\widetilde{y}), \quad k=1,2,3, \\
& y=\widetilde{y}+\Lambda(\widetilde{y}) \eta, \quad \nabla_{y}=\Lambda(\widetilde{y}) \nabla_{\eta}, \\
& \widetilde{a}_{k l}(\widetilde{y})=\lambda_{p k}(\widetilde{y}) a_{p q}(\widetilde{y}) \lambda_{q l}(\widetilde{y})=\left\{\Lambda^{\top}(\widetilde{y}) \mathbf{a}(\widetilde{y}) \Lambda(\widetilde{y})\right\}_{k l}, \\
& \widetilde{\beta}(\widetilde{y})=\frac{1}{3}\left[\widetilde{a}_{11}(\widetilde{y})+\widetilde{a}_{22}(\widetilde{y})+\widetilde{a}_{33}(\widetilde{y})\right] .
\end{aligned}
$$

Here $\widetilde{a}_{k l}(\widetilde{y})$ are the "frozen" coefficients of the principal part of the differential operator $A\left(y, \partial_{y}\right)$ subjected to above described orthogonal transformation related to the local coordinate system and evidently the matrix $\widetilde{\mathbf{a}}(\widetilde{y})=\left[\widetilde{a}_{k l}(\widetilde{y})\right]_{3 \times 3}:=\Lambda^{\top}(\widetilde{y}) \mathbf{a}(\widetilde{y}) \Lambda(\widetilde{y})$ is positive definite, since $\mathbf{a}(\widetilde{y})$ is positive definite (see $(2.1))$. In particular, for arbitrary $\widetilde{y} \in S$ we have

$$
\begin{aligned}
\widetilde{\beta}(\widetilde{y}) & >0, \\
\widetilde{a}_{33}(\widetilde{y}) & =\lambda_{p 3}(\widetilde{y}) a_{p q}(\widetilde{y}) \lambda_{q 3}(\widetilde{y})=a_{p q}(\widetilde{y}) n_{p}(\widetilde{y}) n_{q}(\widetilde{y})=2 \mu(\widetilde{y})>0, \\
T\left(\widetilde{y}, \partial_{y}\right) & =a_{k l}(\widetilde{y}) n_{k}(\widetilde{y}) \partial_{y_{k}}=n(\widetilde{y}) \mathbf{a}(\widetilde{y}) \nabla_{y} \\
& =n(\widetilde{y}) \mathbf{a}(\widetilde{y}) \Lambda(\widetilde{y}) \nabla_{\eta}=n_{p}(\widetilde{y}) a_{p l}(\widetilde{y}) \lambda_{l q}(\widetilde{y}) \partial_{\eta_{q}}
\end{aligned}
$$




$$
=-\lambda_{p 3}(\widetilde{y}) a_{p l}(\widetilde{y}) \lambda_{l q}(\widetilde{y}) \partial_{\eta_{q}}=-\widetilde{a}_{3 q}(\widetilde{y}) \partial_{\eta_{q}},
$$

due to (2.2), (5.9)-(5.11), and (2.33).

Let us show that the Šapiro-Lopatinskii condition (5.8) holds in our case. To this end, we note that the principal homogeneous symbols $\mathfrak{S}_{0}(\tilde{\mathbf{N}})$, $\mathfrak{S}_{0}(\widetilde{\mathcal{B}}), \mathfrak{S}_{0}(\widetilde{\mathbf{P}})$, and $\mathfrak{S}_{0}(\widetilde{\mathcal{V}})$ in the local co-ordinate system with the origin at $\widetilde{y}$, involved in formula (5.8), read as (see (4.5), (4.8), (4.2), (B.31)):

$$
\begin{aligned}
& \mathfrak{S}_{0}(\tilde{\mathbf{N}})(\widetilde{y}, \xi)=\frac{\widetilde{A}(\widetilde{y}, \xi)-\widetilde{\beta}|\xi|^{2}}{|\xi|^{2}}=\frac{\widetilde{a}_{k l}(\widetilde{y}) \xi_{k} \xi_{l}-\widetilde{\beta}|\xi|^{2}}{|\xi|^{2}}, \\
& \mathfrak{S}_{0}(\widetilde{\mathcal{B}})(\widetilde{y}, \xi)=\frac{\widetilde{A}(\widetilde{y}, \xi)}{|\xi|^{2}}=\frac{\widetilde{a}_{k l}(\widetilde{y}) \xi_{k} \xi_{l}}{|\xi|^{2}}, \\
& \mathfrak{S}_{0}(\widetilde{\mathbf{P}})(\xi)=-\frac{1}{|\xi|^{2}}, \quad \mathfrak{S}_{0}(\widetilde{\mathcal{V}})\left(\xi^{\prime}\right)=\frac{1}{2\left|\xi^{\prime}\right|} .
\end{aligned}
$$

As we have mentioned, in $(5.8)$ the functions $\mathfrak{S}_{0}^{(+)}(\widetilde{\mathcal{B}})$ and $\mathfrak{S}_{0}^{(-)}(\widetilde{\mathcal{B}})$ are the "plus" and "minus" factors in the factorization of the symbol $\mathfrak{S}_{0}(\widetilde{\mathcal{B}})$ with respect to the variable $\xi_{3}$, i.e.

$$
\mathfrak{S}_{0}(\widetilde{\mathcal{B}})=\mathfrak{S}_{0}^{(+)}(\widetilde{\mathcal{B}}) \mathfrak{S}_{0}^{(-)}(\widetilde{\mathcal{B}})
$$

where

$$
\mathfrak{S}_{0}^{( \pm)}(\widetilde{\mathcal{B}})(\widetilde{y}, \xi)=\frac{\widetilde{A}^{( \pm)}(\widetilde{y}, \xi)}{\Theta^{( \pm)}(\xi)}
$$

due to (5.18). Here $\Theta^{( \pm)}(\xi)$ and $\widetilde{A}^{( \pm)}(\widetilde{y}, \xi)$ are the "plus" and "minus" factors of the symbols $\Theta(\xi):=|\xi|^{2}=\xi_{1}^{2}+\xi_{2}^{2}+\xi_{3}^{2}=\Theta^{(+)}(\xi) \Theta^{(-)}(\xi)$ and $\widetilde{A}(\widetilde{y}, \xi):=$ $\widetilde{a}_{k l}(\widetilde{y}) \xi_{k} \xi_{l}=\widetilde{A}^{(+)}(\widetilde{y}, \xi) \widetilde{A}^{(-)}(\widetilde{y}, \xi)$, respectively, and

$$
\begin{aligned}
& \Theta^{( \pm)}(\xi):=\left(\xi_{3} \pm i\left|\xi^{\prime}\right|\right), \\
& A^{(+)}(\widetilde{y}, \xi):=\widetilde{a}_{33}(\widetilde{y})\left[\xi_{3}-\tau_{+}\left(\widetilde{y}, \xi^{\prime}\right)\right], A^{(-)}(\widetilde{y}, \xi):=\xi_{3}-\tau_{-}\left(\widetilde{y}, \xi^{\prime}\right) .
\end{aligned}
$$

Here $\tau_{ \pm}\left(\widetilde{y}, \xi^{\prime}\right)$ are the roots of the equation $\widetilde{A}(\widetilde{y}, \xi) \equiv \widetilde{a}_{k l}(\widetilde{y}) \xi_{k} \xi_{l}=0$ with respect to $\xi_{3}$,

$$
\begin{aligned}
& \tau_{+}\left(\widetilde{y}, \xi^{\prime}\right)=\alpha\left(\widetilde{y}, \xi^{\prime}\right)-i \gamma\left(\widetilde{y}, \xi^{\prime}\right), \\
& \tau_{-}\left(\widetilde{y}, \xi^{\prime}\right)=\overline{\tau_{+}\left(\widetilde{y}, \xi^{\prime}\right)}=\alpha\left(\widetilde{y}, \xi^{\prime}\right)+i \gamma\left(\widetilde{y}, \xi^{\prime}\right), \quad \gamma\left(\widetilde{y}, \xi^{\prime}\right)>0, \quad \xi^{\prime} \neq 0 .
\end{aligned}
$$

In what follows, to simplify the notation, we omit the fixed argument $\widetilde{y}$, when it does not lead to misunderstanding. Therefore (5.8) in view of (5.16)-(5.18), (5.19)-(5.20), and (B.31) can be rewritten as

$$
\begin{aligned}
e\left(\xi^{\prime}\right) & =-\Pi^{\prime}\left[\left(\frac{\widetilde{A}-\widetilde{\beta} \Theta}{\Theta} \frac{\Theta^{(+)}}{\widetilde{A}^{(+)}}\right) \Pi^{+}\left(-\frac{1}{\Theta} \frac{\Theta^{(-)}}{\widetilde{A}^{(-)}}\right)\right]\left(\xi^{\prime}\right)-\mathfrak{S}_{0}(\widetilde{\mathcal{V}})\left(\xi^{\prime}\right) \\
& =\Pi^{\prime}\left[\left(\frac{\widetilde{A}-\beta \Theta}{\Theta^{(-)} \widetilde{A}^{(+)}}\right) \Pi^{+}\left(\frac{1}{\Theta^{(+)} \widetilde{A}^{(-)}}\right)\right]\left(\xi^{\prime}\right)-\mathfrak{S}_{0}(\widetilde{\mathcal{V}})\left(\xi^{\prime}\right) \\
& =e^{(1)}\left(\xi^{\prime}\right)+e^{(2)}\left(\xi^{\prime}\right)-\frac{1}{2\left|\xi^{\prime}\right|}
\end{aligned}
$$


where

$$
\begin{aligned}
& e^{(1)}\left(\xi^{\prime}\right)=\Pi^{\prime}\left[\left(\frac{\widetilde{A}^{(-)}}{\Theta^{(-)}}\right) \Pi^{+}\left(\frac{1}{\Theta^{(+)} \widetilde{A}^{(-)}}\right)\right]\left(\xi^{\prime}\right), \\
& e^{(2)}\left(\xi^{\prime}\right)=-\widetilde{\beta} \Pi^{\prime}\left[\left(\frac{\Theta^{(+)}}{\widetilde{A}^{(+)}}\right) \Pi^{+}\left(\frac{1}{\Theta^{(+)} \widetilde{A}^{(-)}}\right)\right]\left(\xi^{\prime}\right) .
\end{aligned}
$$

Applying formula (5.4) along with the residue theorem at $\zeta=-i\left|\xi^{\prime}\right|$, we have,

$$
\begin{aligned}
\Pi^{+}\left(\frac{1}{\Theta^{(+)} \widetilde{A}^{(-)}}\right)(\xi) & =\frac{i}{2 \pi} \lim _{t \rightarrow 0+} \int_{-\infty}^{+\infty} \frac{d \eta_{3}}{\Theta^{(+)}\left(\xi^{\prime}, \eta_{3}\right) \widetilde{A}^{(-)}\left(\xi^{\prime}, \eta_{3}\right)\left(\xi_{3}+i t-\eta_{3}\right)} \\
& =\frac{i}{2 \pi} \lim _{t \rightarrow 0+} \int_{-\infty}^{+\infty} \frac{d \eta_{3}}{\left(\eta_{3}+i\left|\xi^{\prime}\right|\right)\left[\eta_{3}-\tau_{-}\left(\xi^{\prime}\right)\right]\left(\xi_{3}+i t-\eta_{3}\right)} \\
& =-\frac{i}{2 \pi} \lim _{t \rightarrow 0+} \int_{\Gamma^{-}} \frac{d \zeta}{\left(\zeta+i\left|\xi^{\prime}\right|\right)\left[\zeta-\tau_{-}\left(\xi^{\prime}\right)\right]\left(\xi_{3}+i t-\zeta\right)} \\
& =-\frac{i}{2 \pi} \lim _{t \rightarrow 0+} \frac{2 \pi i}{\left[-i\left|\xi^{\prime}\right|-\tau_{-}\left(\xi^{\prime}\right)\right]\left(\xi_{3}+i t+i\left|\xi^{\prime}\right|\right)} \\
& =-\frac{1}{\left[i\left|\xi^{\prime}\right|+\tau_{-}\left(\xi^{\prime}\right)\right]\left(\xi_{3}+i\left|\xi^{\prime}\right|\right)} .
\end{aligned}
$$

Substituting (5.25) into (5.23), employing representation (5.7) and calculating the residue at $\zeta=-i\left|\xi^{\prime}\right|$, we find,

$$
e^{(1)}\left(\xi^{\prime}\right)=\frac{1}{2 \pi} \int_{\Gamma^{-}} \frac{\zeta-\tau_{-}\left(\xi^{\prime}\right)}{\zeta-i\left|\xi^{\prime}\right|} \frac{d \zeta}{\left[i\left|\xi^{\prime}\right|+\tau_{-}\left(\xi^{\prime}\right)\right]\left(\zeta+i\left|\xi^{\prime}\right|\right)}=\frac{1}{2\left|\xi^{\prime}\right|}
$$

Quite similarly, substituting (5.25) into (5.24) and calculating the residue at $\zeta=\tau_{+}\left(\xi^{\prime}\right)$, we get

$$
\begin{aligned}
e^{(2)}\left(\xi^{\prime}\right) & =-\frac{\widetilde{\beta}}{2 \pi} \int_{\Gamma^{-}} \frac{\zeta+i\left|\xi^{\prime}\right|}{\widetilde{a}_{33}\left[\zeta-\tau_{+}\left(\xi^{\prime}\right)\right]} \frac{d \zeta}{\left[i\left|\xi^{\prime}\right|+\tau_{-}\left(\xi^{\prime}\right)\right]\left(\zeta+i\left|\xi^{\prime}\right|\right)} \\
& =-\frac{i \widetilde{\beta}}{\widetilde{a}_{33}\left[i\left|\xi^{\prime}\right|+\tau_{-}\left(\xi^{\prime}\right)\right]} .
\end{aligned}
$$

Substituting to $(5.22)$ we finally obtain

$$
e\left(\xi^{\prime}\right)=-\frac{i \widetilde{\beta}}{\widetilde{a}_{33}\left[i\left|\xi^{\prime}\right|+\tau_{-}\left(\xi^{\prime}\right)\right]}=-\frac{\widetilde{\beta}}{\widetilde{a}_{33}} \frac{\gamma\left(\xi^{\prime}\right)+\left|\xi^{\prime}\right|+i \alpha\left(\xi^{\prime}\right)}{\alpha^{2}\left(\xi^{\prime}\right)+\left(\gamma\left(\xi^{\prime}\right)+\left|\xi^{\prime}\right|\right)^{2}} .
$$

By (5.13), (5.14), and (5.21) we then deduce $\operatorname{Re} e\left(\xi^{\prime}\right)<0$ for all $\xi^{\prime} \neq 0$. Thus, for the operator $\mathfrak{D}$ the Šapiro-Lopatinskii condition holds. Therefore operator (5.3) is Fredholm for $r>-1 / 2$.

Now we will show that index of the operator $\mathfrak{D}$ equals to zero. To this end let us consider the operator

$$
\mathfrak{D}_{t}:=\left[\begin{array}{cc}
\mathcal{B} & -r_{\Omega^{+}} V \\
t \mathbf{N}^{+} E_{0} & -\mathcal{V}
\end{array}\right]
$$


with $t \in[0,1]$, and establish that it is homotopic to the operator $\mathfrak{D}=\mathfrak{D}_{1}$.

We have to check that for the operator $\mathfrak{D}_{t}$ the Šapiro-Lopatinskii condition is satisfied as well. In this case, for the corresponding boundary symbol we have the following expression, cf. (5.8),

$$
\begin{aligned}
e_{t}\left(\xi^{\prime}\right) & =-\Pi^{\prime}\left[\frac{\mathfrak{S}_{0}(t \widetilde{\mathbf{N}})}{\mathfrak{S}_{0}^{(+)}(\widetilde{\mathcal{B}})} \Pi^{+}\left(\frac{\mathfrak{S}_{0}(\widetilde{\mathbf{P}})}{\mathfrak{S}_{0}^{(-)}(\widetilde{\mathcal{B}})}\right)\right]\left(\xi^{\prime}\right)-\mathfrak{S}_{0}(\widetilde{\mathcal{V}})\left(\xi^{\prime}\right) \\
& =t\left[e^{(1)}\left(\xi^{\prime}\right)+e^{(2)}\left(\xi^{\prime}\right)\right]-\frac{1}{2\left|\xi^{\prime}\right|}=t e\left(\xi^{\prime}\right)+\frac{t-1}{2\left|\xi^{\prime}\right|} \\
& =\frac{t-1}{2\left|\xi^{\prime}\right|}-\frac{t \widetilde{\beta}}{\widetilde{a}_{33}} \frac{\gamma\left(\xi^{\prime}\right)+\left|\xi^{\prime}\right|+i \alpha\left(\xi^{\prime}\right)}{\alpha^{2}\left(\xi^{\prime}\right)+\left(\gamma\left(\xi^{\prime}\right)+\left|\xi^{\prime}\right|\right)^{2}} .
\end{aligned}
$$

Since $\widetilde{\beta}>0, \gamma\left(\xi^{\prime}\right)>0, \widetilde{a}_{33}>0$, and $t \in[0,1]$, we infer

$$
\operatorname{Re} e_{t}\left(\xi^{\prime}\right)=\frac{t-1}{2\left|\xi^{\prime}\right|}-\frac{t \widetilde{\beta}}{\widetilde{a}_{33}} \frac{\gamma\left(\xi^{\prime}\right)+\left|\xi^{\prime}\right|}{\alpha^{2}\left(\xi^{\prime}\right)+\left(\gamma\left(\xi^{\prime}\right)+\left|\xi^{\prime}\right|\right)^{2}}<0 \text { for all } \xi^{\prime} \neq 0,
$$

which implies that for the operator $\mathfrak{D}_{t}$ the Šapiro-Lopatinskii condition is satisfied. Therefore the operator

$$
\mathfrak{D}_{t}: H^{r+1}\left(\Omega^{+}\right) \times H^{r-\frac{1}{2}}(S) \rightarrow H^{r+1}\left(\Omega^{+}\right) \times H^{r+\frac{1}{2}}(S)
$$

is Fredholm and thus has the same index for all $r>-1 / 2$ and for all $t \in[0,1]$ (see, e.g., [26, Chapter 1, Theorem 3.11]).

On the other hand, the upper triangular matrix operator $\mathfrak{D}_{0}$ has zero index since the operators on the main diagonal, $\mathcal{B}$ and $\mathcal{V}$, are Fredholm with zero index in appropriate function spaces. Indeed, for the operator $\mathcal{B}$ it has been shown in Section 4, while for the operator $\mathcal{V}$ it follows from the fact that $\mathcal{V}$ is a compact perturbation of the invertible harmonic single layer operator $([7],[18])$. Consequently, Ind $\mathfrak{D}=\operatorname{Ind} \mathfrak{D}_{1}=\operatorname{Ind} \mathfrak{D}_{t}=\operatorname{Ind} \mathfrak{D}_{0}=0$.

Theorem 5.2. Let a localizing function $\chi \in X_{+}^{\infty}$ and $r>-1 / 2$. Then operator (5.3) is invertible.

Proof. Since by Lemma 5.1 the operator $\mathfrak{D}$ is Fredholm with zero index, its injectivity implies the invertibility. Thus, it remains to show that the null space of the operator $\mathfrak{D}$ is trivial for $r>-1 / 2$.

Assume that $U=(u, \psi)^{\top} \in H^{r+1}\left(\Omega^{+}\right) \times H^{r-\frac{1}{2}}(S)$ is a solution to the homogeneous equation

$$
\mathfrak{D} U=0 .
$$

Since the index of of the operator $\mathfrak{D}$ is zero, there exists its left regularizer,

$$
\mathfrak{L}: H^{r+1}\left(\Omega^{+}\right) \times H^{r+\frac{1}{2}}(S) \rightarrow H^{r+1}\left(\Omega^{+}\right) \times H^{r-\frac{1}{2}}(S),
$$

such that $\mathfrak{L} \mathfrak{D}=I+\mathfrak{T}$, where $\mathfrak{T}$ is the operator of order -1 (cf. proofs of Theorems 22.1 and 23.1 in [15]),

$$
\mathfrak{T}: H^{r+1}\left(\Omega^{+}\right) \times H^{r-\frac{1}{2}}(S) \rightarrow H^{r+2}\left(\Omega^{+}\right) \times H^{r+\frac{1}{2}}(S) .
$$


Therefore, from (5.26) we have

$$
\mathfrak{L} \mathfrak{D} U=U+\mathfrak{T} U=0 .
$$

From (5.27) we see that $\mathfrak{T} U \in H^{r+2}\left(\Omega^{+}\right) \times H^{r+\frac{1}{2}}(S)$, and consequently, by (5.28) we get

$$
U=(u, \psi)^{\top} \in H^{r+2}\left(\Omega^{+}\right) \times H^{r+\frac{1}{2}}(S) .
$$

If $r \geq 0$, this implies $u \in H^{1,0}\left(\Omega^{+} ; A\right)$. If $-1 / 2<r<0$, we iterate the above reasoning for $U$ satisfying (5.29) to arrive at the inclusion $U=(u, \psi)^{\top} \in$ $H^{r+3}\left(\Omega^{+}\right) \times H^{r+\frac{3}{2}}(S)$ which again implies $u \in H^{1,0}\left(\Omega^{+} ; A\right)$. Then we can apply the equivalence Theorem 3.1 to conclude that a solution $U=(u, \psi)^{\top}$ to the homogeneous equation (5.26) is zero vector because $u$ solves the homogeneous Dirichlet problem and the relation $T^{+} u=\psi$ holds on $S$. This implies that the null space of $\mathfrak{D}$ is trivial in the class $H^{r+1}\left(\Omega^{+}\right) \times H^{r-\frac{1}{2}}(S)$. Consequently, the operator $\mathfrak{D}: H^{r+1}\left(\Omega^{+}\right) \times H^{r-\frac{1}{2}}(S) \rightarrow H^{r+1}\left(\Omega^{+}\right) \times H^{r+\frac{1}{2}}(S)$ is invertible.

For a localizing function $\chi$ of finite smoothness we have the following result.

Corollary 5.3. If $\chi \in X_{+}^{3}$, then the operator

$$
\mathfrak{D}: H^{1}\left(\Omega^{+}\right) \times H^{-\frac{1}{2}}(S) \rightarrow H^{1}\left(\Omega^{+}\right) \times H^{\frac{1}{2}}(S)
$$

is invertible.

Proof. It can be carried out by the word for word arguments applied in the proofs of Lemmas 5.1 and 4.1, Theorem 5.2 with $r=0$, and using Theorems B.3 and B.4 that provide mapping properties of the localized potentials for a localizing function of finite smoothness.

The following result follows immediately from Lemma 2.2 and Corollaries 5.3 and 2.3 .

Corollary 5.4. If $\chi \in X_{+}^{3}$, then the operator

$$
\mathfrak{D}: H^{1,0}\left(\Omega^{+}, A\right) \times H^{-\frac{1}{2}}(S) \rightarrow H^{1,0}\left(\Omega^{+} ; \Delta\right) \times H^{\frac{1}{2}}(S)
$$

is invertible.

\section{Invertibility of the LBDSIO for the Robin problem}

From Theorem 3.3 it follows that LBDSIE system (3.12)-(3.13), which has a special right-hand side, is uniquely solvable in the class $H^{1,0}\left(\Omega^{+} ; A\right) \times H^{\frac{1}{2}}(S)$. However, as in the previous case, this fact does not imply invertibility of the LBDSIO generated by the left-hand side expressions in (3.12)-(3.13). In what follows, we investigate Fredholm properties of this operator and establish invertibility results in appropriate function spaces.

To this end, we consider the LBDSIE system

$$
[\beta \mathbf{I}+\mathbf{N}] E_{0} u+W \varphi+V M_{\kappa} \varphi=F_{1} \text { in } \Omega^{+},
$$




$$
\mathbf{N}^{+} E_{0} u+(\beta-\mu) \varphi+\mathcal{W} \varphi+\mathcal{V} M_{\kappa} \varphi=F_{2} \text { on } S,
$$

where $F_{1} \in H^{1}\left(\Omega^{+}\right), F_{2} \in H^{\frac{1}{2}}(S), \kappa$ is a smooth nonnegative function involved in the Robin boundary condition (2.9), and $M_{\kappa}$ is the multiplication operator, $\left(M_{\kappa} \varphi\right)(x):=\kappa(x) \varphi(x)$. Denote by $\mathfrak{R}$ the localized boundarydomain integral operator generated by the left-hand side expressions in LBDSIE system (6.1)-(6.2),

$$
\mathfrak{R}:=\left[\begin{array}{ll}
r_{\Omega^{+}}(\beta \mathbf{I}+\mathbf{N}) E_{0} & r_{\Omega^{+}}\left[W+V M_{\kappa}\right] \\
\mathbf{N}^{+} E_{0} & (\beta-\mu) \mathbf{I}+\mathcal{W}+\mathcal{V} M_{\kappa}
\end{array}\right] .
$$

Lemma 6.1. Let the localizing function $\chi \in X^{\infty}$ and $r>-1 / 2$. Then the operator

$$
\mathfrak{R}: H^{r+1}\left(\Omega^{+}\right) \times H^{r+\frac{1}{2}}(S) \rightarrow H^{r+1}\left(\Omega^{+}\right) \times H^{r+\frac{1}{2}}(S)
$$

is Fredholm with zero index.

Proof. As we mentioned in the proof of Lemma 5.1, if a localizing function is infinitely differentiable, $\chi \in X_{+}^{\infty}$, then the localized volume and layer potentials and the corresponding boundary operators have exactly the same mapping properties as the corresponding harmonic potentials (see, e.g., [6, Ch. 5, Theorem 2.4(i)], [18, Theorem 8.4.13], [27, Ch. 2]), which implies that the operator (6.4) is continuous for all $r>-1 / 2$ if $\chi \in X^{\infty}$. Further, we note that the operators

$$
V: H^{r+\frac{1}{2}}(S) \rightarrow H^{r+1}\left(\Omega^{+}\right), \quad \mathcal{V}: H^{r+\frac{1}{2}}(S) \rightarrow H^{r+\frac{1}{2}}(S)
$$

are compact since they have the following mapping properties

$$
V: H^{r+\frac{1}{2}}(S) \rightarrow H^{r+2}\left(\Omega^{+}\right), \quad \mathcal{V}: H^{r+\frac{1}{2}}(S) \rightarrow H^{r+3 / 2}(S), \quad r>-\frac{1}{2} .
$$

To prove the lemma we apply the same scheme as in the proof of Lemma 5.1. We have to show that the Sapiro-Lopatinskii condition for the operator $\mathfrak{R}$ is satisfied (cf. [15], Theorem 12.2). To this end, let us take into consideration formulas (5.9)-(5.15) and arguments in the proof of Lemma 5.1 related to local coordinate systems, the expressions for the principal homogeneous symbols of the operators $\beta \mathbf{I}+\mathbf{N}$ and $\mathbf{N}$, representation formula (B.8) of the double layer potential operator, and also Remark B.7 for the principal homogeneous symbol of the operator $\mathcal{W}$.

Here we employ the notation in Section 5 and on the basis of the above mentioned relations we construct the following matrix associated with principal homogeneous symbols of the operators involved in (6.3) at some local co-ordinate system with the origin at a point $\widetilde{y} \in S$ :

$$
\mathbf{R}(\widetilde{y}, \xi):=\left[\begin{array}{ll}
R_{11}(\widetilde{y}, \xi) & R_{12}(\widetilde{y}, \xi) \\
R_{21}(\widetilde{y}, \xi) & R_{22}\left(\widetilde{y}, \xi^{\prime}\right)
\end{array}\right],
$$

where

$$
R_{11}(\widetilde{y}, \xi):=\mathfrak{S}_{0}(\widetilde{\mathcal{B}})(\widetilde{y}, \xi) \equiv \mathfrak{S}_{0}(\widetilde{\beta} \mathbf{I}+\widetilde{\mathbf{N}})(\widetilde{y}, \xi)
$$




$$
\begin{aligned}
&=\frac{\widetilde{A}(\xi)}{|\xi|^{2}}=\frac{\widetilde{a}_{k l}(\widetilde{y}) \xi_{k} \xi_{l}}{|\xi|^{2}}>0, \\
& R_{12}(\widetilde{y}, \xi):=\mathfrak{S}_{0}\left(\widetilde{a}_{k l}(\widetilde{y}) n_{k}(\widetilde{y}) \partial_{l} \widetilde{\mathbf{P}}\right)=-\frac{\widetilde{a}_{3 l}(\widetilde{y})\left(i \xi_{l}\right)}{|\xi|^{2}}, \\
& R_{21}(\widetilde{y}, \xi):=\mathfrak{S}_{0}(\widetilde{\mathbf{N}})(\widetilde{y}, \xi)=\frac{\widetilde{A}(\xi)}{|\xi|^{2}}-\widetilde{\beta}=\frac{\widetilde{a}_{k l}(\widetilde{y}) \xi_{k} \xi_{l}-\widetilde{\beta}|\xi|^{2}}{|\xi|^{2}}, \\
& R_{22}\left(\widetilde{y}, \xi^{\prime}\right):=\mathfrak{S}_{0}((\widetilde{\beta}-\widetilde{\mu}) \mathbf{I}+\widetilde{\mathcal{W}})\left(\widetilde{y}, \xi^{\prime}\right) \\
&=\frac{1}{2}\left[\left[2 \widetilde{\beta}-\widetilde{a}_{33}(\widetilde{y})\right]-i \sum_{l=1}^{2} \widetilde{a}_{3 l}(\widetilde{y}) \frac{\xi_{l}}{\left|\xi^{\prime}\right|}\right], \\
& \widetilde{\mu}:=\mu(\widetilde{y}), \quad \xi=\left(\xi_{1}, \xi_{2}, \xi_{3}\right) \in \mathbb{R}^{3} \backslash\{0\}, \quad \xi^{\prime}=\left(\xi_{1}, \xi_{2}\right) \in \mathbb{R}^{2} \backslash\{0\} .
\end{aligned}
$$

Recall that summation over repeated indices is assumed from 1 to 3 if not otherwise stated and the outward unit normal vector $n(\widetilde{y})$ has the components $(0,0,-1)^{\top}$ in the chosen local coordinate system.

The Šapiro-Lopatinskii condition then takes the form (see [15], Theorem 12.2 , formulas $(12.25)$ and $(12.27))$

$$
\begin{array}{r}
e\left(\widetilde{y}, \xi^{\prime}\right)=-\Pi^{\prime}\left[\frac{R_{21}(\widetilde{y}, \cdot)}{R_{11}^{(+)}(\widetilde{y}, \cdot)} \Pi^{+}\left(\frac{R_{12}(\widetilde{y}, \cdot)}{R_{11}^{(-)}(\widetilde{y}, \cdot)}\right)\right]\left(\xi^{\prime}\right)+R_{22}\left(\widetilde{y}, \xi^{\prime}\right) \neq 0, \\
\forall \xi^{\prime} \in \mathbb{R}^{2} \backslash\{0\}, \quad \forall \widetilde{y} \in \partial \Omega,
\end{array}
$$

where $\Pi^{+}$and $\Pi^{\prime}$ are defined in (5.4) and (5.5), while

$$
\begin{aligned}
& R_{11}(\widetilde{y}, \xi)=R_{11}^{(+)}(\widetilde{y}, \xi) R_{11}^{(-)}(\widetilde{y}, \xi), \\
& R_{11}^{(+)}(\widetilde{y}, \xi)=\frac{\widetilde{A}^{(+)}(\widetilde{y}, \xi)}{\Theta^{(+)}(\xi)}=\frac{\widetilde{a}_{33}(\widetilde{y})\left[\xi_{3}-\tau_{+}\left(\widetilde{y}, \xi^{\prime}\right)\right]}{\xi_{3}+i\left|\xi^{\prime}\right|}, \\
& R_{11}^{(-)}(\widetilde{y}, \xi)=\frac{\widetilde{A}^{(-)}(\widetilde{y}, \xi)}{\Theta^{(-)}(\xi)}=\frac{\xi_{3}-\tau_{-}\left(\widetilde{y}, \xi^{\prime}\right)}{\xi_{3}-i\left|\xi^{\prime}\right|}
\end{aligned}
$$

and the notations (5.19)-(5.21) are employed.

As in the proof of Lemma 5.1, we will further drop, when possible, the fixed argument $\widetilde{y}$, associated with the origin of the local coordinate system.

Let $\vartheta\left(\xi^{\prime}\right):=\sum_{l=1}^{2} \widetilde{a}_{3 l} \xi_{l}$. It is easy to see that

$$
2 \vartheta\left(\xi^{\prime}\right)=-\widetilde{a}_{33}\left[\tau_{+}\left(\xi^{\prime}\right)+\tau_{-}\left(\xi^{\prime}\right)\right]
$$

since $\tau_{+}$and $\tau_{-}$are roots of the quadratic equation

$$
\widetilde{A}(\xi)=\widetilde{a}_{33} \xi_{3}^{2}+2 \vartheta\left(\xi^{\prime}\right) \xi_{3}+\sum_{k, l=1}^{2} \widetilde{a}_{k l} \xi_{k} \xi_{l}=0 .
$$

From (5.4), calculating the residue at $\eta_{3}=-i\left|\xi^{\prime}\right|$, we have,

$$
\Pi^{+}\left(\frac{R_{12}}{R_{11}^{(-)}}\right)(\xi)=\Pi^{+}\left(\frac{-i \widetilde{a}_{3 l} \xi_{l}}{|\xi|^{2}} \frac{\xi_{3}-i\left|\xi^{\prime}\right|}{\xi_{3}-\tau_{-}}\right)\left(\xi^{\prime}\right)
$$




$$
\begin{aligned}
& =\Pi^{+}\left(\frac{-i \widetilde{a}_{3 l} \xi_{l}}{\xi_{3}+i\left|\xi^{\prime}\right|} \frac{1}{\xi_{3}-\tau_{-}}\right)\left(\xi^{\prime}\right) \\
& =\frac{i}{2 \pi} \lim _{t \rightarrow 0+} \int_{-\infty}^{+\infty} \frac{\left[-i \vartheta\left(\xi^{\prime}\right)-i \widetilde{a}_{33} \eta_{3}\right] d \eta_{3}}{\left(\eta_{3}+i\left|\xi^{\prime}\right|\right)\left(\eta_{3}-\tau_{-}\right)\left(\xi_{3}+i t-\eta_{3}\right)} \\
& =\frac{i \vartheta\left(\xi^{\prime}\right)+\widetilde{a}_{33}\left|\xi^{\prime}\right|}{\left(\tau_{-}+i\left|\xi^{\prime}\right|\right)\left(\xi_{3}+i\left|\xi^{\prime}\right|\right)} .
\end{aligned}
$$

Further, calculating residues at $\xi_{3}=-\left|\xi^{\prime}\right|$ and $\xi_{3}=\tau_{+}$, we find

$$
\begin{aligned}
\Pi^{\prime} & {\left[\frac{R_{21}}{R_{11}^{(+)}} \Pi^{+}\left(\frac{R_{12}}{R_{11}^{(-)}}\right)\right]\left(\xi^{\prime}\right) } \\
& =-\frac{1}{2 \pi} \int_{\Gamma^{-}}\left(\frac{\widetilde{A}(\xi)}{|\xi|^{2}}-\widetilde{\beta}\right) \frac{\xi_{3}+i\left|\xi^{\prime}\right|}{\widetilde{a}_{33}\left(\xi_{3}-\tau_{+}\right)} \frac{i \vartheta\left(\xi^{\prime}\right)+\widetilde{a}_{33}\left|\xi^{\prime}\right|}{\left(\tau_{-}+i\left|\xi^{\prime}\right|\right)\left(\xi_{3}+i\left|\xi^{\prime}\right|\right)} d \xi_{3} \\
& =-\frac{1}{2 \pi} \frac{i \vartheta\left(\xi^{\prime}\right)+\widetilde{a}_{33}\left|\xi^{\prime}\right|}{\tau_{-}+i\left|\xi^{\prime}\right|} \int_{\Gamma^{-}}\left[\frac{\xi_{3}-\tau_{-}}{\xi_{3}^{2}+\left|\xi^{\prime}\right|^{2}}-\frac{\widetilde{\beta}}{\widetilde{a}_{33}\left(\xi_{3}-\tau_{+}\right)}\right] d \xi_{3} \\
& =-\frac{i \vartheta\left(\xi^{\prime}\right)+\widetilde{a}_{33}\left|\xi^{\prime}\right|}{2\left|\xi^{\prime}\right|}+\frac{i \widetilde{\beta}}{\widetilde{a}_{33}} \frac{i \vartheta\left(\xi^{\prime}\right)+\widetilde{a}_{33}\left|\xi^{\prime}\right|}{\tau_{-}+i\left|\xi^{\prime}\right|} .
\end{aligned}
$$

Now, from (6.10) and (6.6)-(6.9) we get

$$
\begin{aligned}
e\left(\xi^{\prime}\right) & =\frac{i \vartheta\left(\xi^{\prime}\right)+\widetilde{a}_{33}\left|\xi^{\prime}\right|}{2\left|\xi^{\prime}\right|}-\frac{i \widetilde{\beta}}{\widetilde{a}_{33}} \frac{i \vartheta\left(\xi^{\prime}\right)+\widetilde{a}_{33}\left|\xi^{\prime}\right|}{\tau_{-}\left(\xi^{\prime}\right)+i\left|\xi^{\prime}\right|}+\frac{1}{2}\left[2 \widetilde{\beta}-\widetilde{a}_{33}-i \frac{\vartheta\left(\xi^{\prime}\right)}{\left|\xi^{\prime}\right|}\right] \\
& =\frac{\widetilde{\beta}\left[\vartheta\left(\xi^{\prime}\right)+\widetilde{a}_{33} \tau_{-}\left(\xi^{\prime}\right)\right]}{\widetilde{a}_{33}\left[\tau_{-}\left(\xi^{\prime}\right)+i\left|\xi^{\prime}\right|\right]} .
\end{aligned}
$$

Finally, in view of $(5.11),(6.11)$, and $(5.21)$, we arrive at the relation

$$
e\left(\xi^{\prime}\right)=\frac{\widetilde{\beta}\left[\tau_{-}\left(\xi^{\prime}\right)-\tau_{+}\left(\xi^{\prime}\right)\right]}{2\left[\tau_{-}\left(\xi^{\prime}\right)+i\left|\xi^{\prime}\right|\right]}=\frac{i \widetilde{\beta} \gamma\left(\xi^{\prime}\right)}{\tau_{-}\left(\xi^{\prime}\right)+i\left|\xi^{\prime}\right|} \neq 0 \text { for } \xi^{\prime} \neq 0,
$$

which shows that the Šapiro-Lopatinskii condition for the operator $\mathfrak{R}$ is fulfilled. Thus the operator $\mathfrak{R}$ in (6.4) if Fredholm.

With the help of the homotopy principle as in the proof of Lemma 5.1, we now show that Ind $\mathfrak{R}=0$. To this end we consider the operator

$$
\mathfrak{R}_{t}:=\left[\begin{array}{ll}
r_{\Omega^{+}}(\beta \mathbf{I}+\mathbf{N}) E_{0} & r_{\Omega^{+}}\left[W+V M_{\kappa}\right] \\
t \mathbf{N}^{+} E_{0} & \beta \mathbf{I}+t\left(-\mu \mathbf{I}+\mathcal{W}+\mathcal{V} M_{\kappa}\right)
\end{array}\right]
$$

with $t \in[0,1]$ and show that it is homotopic to the operator $\mathfrak{R}=\mathfrak{R}_{1}$. The operator $\mathfrak{R}_{t}$ is evidently continuous in $t \in[0,1]$. Moreover, it is also Fredholm operator for each $t \in[0,1]$. Indeed, the boundary symbol corresponding to (6.13) now reads as (cf. (6.5), (6.10), and (6.13))

$$
e_{t}\left(\xi^{\prime}\right)=-\Pi^{\prime}\left[\frac{t R_{21}}{R_{11}^{(+)}} \Pi^{+}\left(\frac{R_{12}}{R_{11}^{(-)}}\right)\right]\left(\xi^{\prime}\right)+\mathfrak{S}_{0}(\widetilde{\beta} \mathbf{I}+t[-\widetilde{\mu} \mathbf{I}+\widetilde{\mathcal{W}}])\left(\xi^{\prime}\right)
$$




$$
\begin{aligned}
& =-\Pi^{\prime}\left[\frac{t R_{21}}{R_{11}^{(+)}} \Pi^{+}\left(\frac{R_{12}}{R_{11}^{(-)}}\right)\right]\left(\xi^{\prime}\right)+t R_{22}\left(\xi^{\prime}\right)+(1-t) \widetilde{\beta} \\
& =t e\left(\xi^{\prime}\right)+(1-t) \widetilde{\beta}
\end{aligned}
$$

where $e\left(\xi^{\prime}\right)$ is defined in (6.12). Since $\widetilde{\beta}>0$ and

$$
\operatorname{Re} e\left(\xi^{\prime}\right)=\frac{\widetilde{\beta} \gamma\left(\xi^{\prime}\right)\left[\gamma\left(\xi^{\prime}\right)+\left|\xi^{\prime}\right|\right]}{\alpha^{2}\left(\xi^{\prime}\right)+\left[\gamma\left(\xi^{\prime}\right)+\left|\xi^{\prime}\right|\right]^{2}}>0 \text { for } \xi^{\prime} \neq 0,
$$

we see that $\operatorname{Re} e_{t}\left(\xi^{\prime}\right)=t \operatorname{Re} e\left(\xi^{\prime}\right)+(1-t) \widetilde{\beta}>0$ for all $\xi^{\prime} \neq 0$ and for all $t \in[0,1]$. Thus, the Sapiro-Lopatinskii condition for the operator $\mathfrak{R}_{t}$ holds for all $t \in[0,1]$ and, consequently, the operator $\Re_{t}: H^{r+1}\left(\Omega^{+}\right) \times H^{r+\frac{1}{2}}(S) \rightarrow$ $H^{r+1}\left(\Omega^{+}\right) \times H^{r+\frac{1}{2}}(S)$ is Fredholm and thus homotopic to $\mathfrak{R}_{1}$.

Hence $\mathfrak{R}_{t}$ has the same index for all $t \in[0,1]$ (see, e.g., [26, Chapter 1, Theorem 3.11]). On the other hand, by Lemma 4.1 it is evident that the index of the upper triangular operator $\mathfrak{R}_{0}$ equals to zero, since the operators in the main diagonal, $\mathcal{B}=r_{\Omega^{+}}(\beta \mathbf{I}+\mathbf{N}) E_{0}$ and $\beta \mathbf{I}$, are Fredholm with zero index. Therefore Ind $\mathfrak{R}=$ Ind $\mathfrak{R}_{1}=$ Ind $\mathfrak{R}_{t}=$ Ind $\mathfrak{R}_{0}=0$.

Theorem 6.2. Let a localizing function $\chi \in X_{+}^{\infty}$ and $r>-1 / 2$. Then the operator

$$
\Re: H^{r+1}\left(\Omega^{+}\right) \times H^{r+\frac{1}{2}}(S) \rightarrow H^{r+1}\left(\Omega^{+}\right) \times H^{r+\frac{1}{2}}(S)
$$

is invertible.

Proof. Continuity of the operator (6.14) directly follows from the mapping properties of the localized potentials associated with an infinitely smooth localizing cut off function (see the paragraph after formula (5.3) in Section $5)$.

Further, let us show that the null space of the operator (6.14) is trivial. Indeed, let a vector $U=(u, \varphi)^{\top} \in H^{r+1}\left(\Omega^{+}\right) \times H^{r+\frac{1}{2}}(S)$ be a solution of the homogeneous equation

$$
\mathfrak{R} U=0 .
$$

Since the operator $\mathfrak{R}$ is Fredholm with zero index there exists a left regularizer $\mathfrak{L}_{1}$ such that

$$
\mathfrak{L}_{1}: H^{r+1}\left(\Omega^{+}\right) \times H^{r+\frac{1}{2}}(S) \rightarrow H^{r+1}\left(\Omega^{+}\right) \times H^{r+\frac{1}{2}}(S)
$$

and $\mathfrak{L}_{1} \mathfrak{R}=I+\mathfrak{T}_{1}$, where $\mathfrak{T}_{1}$ is the operator of order -1 (see [15], Theorems 22.1 and 23.1),

$$
\mathfrak{T}_{1}: H^{r+1}\left(\Omega^{+}\right) \times H^{r+\frac{1}{2}}(S) \rightarrow H^{r+2}\left(\Omega^{+}\right) \times H^{r+3 / 2}(S) .
$$

Therefore, from (6.15) we have

$$
\mathfrak{L}_{1} \mathfrak{R} U=U+\mathfrak{T}_{1} U=0 .
$$

From (6.16), we see that

$$
\mathfrak{T}_{1} U \in H^{r+2}\left(\Omega^{+}\right) \times H^{r+3 / 2}(S),
$$


and, consequently,

$$
U=(u, \psi)^{\top} \in H^{r+2}\left(\Omega^{+}\right) \times H^{r+3 / 2}(S)
$$

due to (6.17). For $r \geq 0$ this implies $u \in H^{1,0}\left(\Omega^{+} ; A\right)$. If $-1 / 2<r<0$, we iterate the above reasoning for $U$ satisfying (6.18) to show that

$$
U=(u, \psi)^{\top} \in H^{r+3}\left(\Omega^{+}\right) \times H^{r+\frac{5}{2}}(S),
$$

which again implies $u \in H^{1,0}\left(\Omega^{+} ; A\right)$. Therefore we can apply the equivalence Theorem 3.3 to conclude that a solution $U=(u, \varphi)^{\top}$ to the homogeneous equation (6.15) is zero vector, since $u$ solves then the homogeneous Robin problem and the relation $\gamma^{+} u=\varphi$ on $S$ holds. Thus Ker $\mathfrak{R}=\{0\}$ in the class $H^{r+1}\left(\Omega^{+}\right) \times H^{r+\frac{1}{2}}(S)$. Then it follows that the operator $(6.14)$ is Fredholm with zero index and injective, and therefore it is invertible.

For a localizing function $\chi$ of finite smoothness we have the following result.

Corollary 6.3. If $\chi \in X_{+}^{3}$, then the operator

$$
\mathfrak{R}: H^{1}\left(\Omega^{+}\right) \times H^{\frac{1}{2}}(S) \rightarrow H^{1}\left(\Omega^{+}\right) \times H^{\frac{1}{2}}(S)
$$

is invertible.

Proof. It can be carried out by the word for word arguments applied in the proof of Theorem 6.2 with $r=0$ and using Theorems B.3, B.4, and B.5 that provide mapping properties of the localized potentials for a localizing function of finite smoothness.

As in the previous section, Lemma 2.2 and Corollaries 6.3 and 2.3 immediately imply the following assertion.

Corollary 6.4. If $\chi \in X_{+}^{3}$, then the operator

$$
\mathfrak{R}: H^{1,0}\left(\Omega^{+} ; A\right) \times H^{\frac{1}{2}}(S) \rightarrow H^{1,0}\left(\Omega^{+} ; \Delta\right) \times H^{\frac{1}{2}}(S)
$$

is invertible.

\section{Fredholm properties of the LBDSIO for the Neumann problem}

The operator generated by the LBDSIE system (3.6) corresponding to the Neumann BVP reads as

$$
\mathfrak{N}:=\left[\begin{array}{ll}
r_{\Omega^{+}}(\beta \mathbf{I}+\mathbf{N}) E_{0} & r_{\Omega^{+}} W \\
\mathbf{N}^{+} E_{0} & (\beta-\mu) \mathbf{I}+\mathcal{W}
\end{array}\right],
$$

and the operators

$$
\begin{aligned}
\mathfrak{N}: H^{r+1}\left(\Omega^{+}\right) \times H^{r+\frac{1}{2}}(S) \rightarrow H^{r+1}\left(\Omega^{+}\right) \times H^{r+\frac{1}{2}}(S), & \\
& \quad \chi \in X_{+}^{\infty}, \quad r>-\frac{1}{2},
\end{aligned}
$$




$$
\begin{aligned}
& \mathfrak{N}: H^{1}\left(\Omega^{+}\right) \times H^{\frac{1}{2}}(S) \rightarrow H^{1}\left(\Omega^{+}\right) \times H^{\frac{1}{2}}(S), \quad \chi \in X_{+}^{3}, \\
& \mathfrak{N}: H^{1,0}\left(\Omega^{+} ; A\right) \times H^{\frac{1}{2}}(S) \rightarrow H^{1,0}\left(\Omega^{+} ; \Delta\right) \times H^{\frac{1}{2}}(S), \quad \chi \in X_{+}^{3},
\end{aligned}
$$

are continuous. Note that the continuity of the above operators still holds if the subscript + is dropped in the classes $X_{+}^{k}$.

In view of (6.3) it is easy to see that the operator $\mathfrak{N}$ is a compact perturbation of the operator $\mathfrak{R}$. Therefore, from the above obtained results for the operator $\mathfrak{R}$, it follows that the operators (7.1) and (7.2) are Fredholm with zero index. Applying the equivalence Theorem 3.2(iii) we see that in $H^{1,0}\left(\Omega^{+} ; A\right) \times H^{\frac{1}{2}}(S)$ and thus in $H^{1}\left(\Omega^{+}\right) \times H^{\frac{1}{2}}(S)$ the null space of the operator is one-dimensional and $\operatorname{Ker} \mathfrak{N}=\left\{c U^{(1)}\right\}$, where $U^{(1)}=(1,1)^{\top}$ and $c$ is an arbitrary constant. Employing the same arguments as in the proofs of Theorems 5.2 and 6.2 we can conclude that the operator $\mathfrak{N}$ in (7.1) with any $r>-1 / 2$ has the same null space $\left\{c U^{(1)}\right\}$. Thus we arrive at the following assertion.

Theorem 7.1. Operators (7.1), (7.2), and (7.3) are Fredholm with zero index and possess a one-dimensional null space $\left\{c U^{(1)}\right\}$, where $U^{(1)}=(1,1)^{\top}$ and $c$ is an arbitrary constant.

\section{APPENDICES}

\section{Appendix A. Classes of localizing functions}

Here we present the classes of localizing functions from [9] used in the main text.

Definition A.1. We say $\chi \in X^{k}$ for integer $k \geq 0$ if $\chi(x)=\breve{\chi}(|x|), \breve{\chi} \in$ $W_{1}^{k}(0, \infty)$ and $\varrho \breve{\chi}(\varrho) \in L_{1}(0, \infty)$.

We say $\chi \in X_{+}^{k}$ for integer $k \geq 1$ if $\chi \in X^{k}, \chi(0)=1$ and

$$
\sigma_{\chi}(\omega)>0 \quad \forall \omega \in \mathbb{R},
$$

where

$$
\sigma_{\chi}(\omega):= \begin{cases}\frac{\hat{\chi}_{s}(\omega)}{\omega} & \text { for } \omega \in \mathbb{R} \backslash\{0\}, \\ \int_{0}^{\infty} \varrho \breve{\chi}(\varrho) d \varrho & \text { for } \omega=0,\end{cases}
$$

and $\hat{\chi}_{s}(\omega)$ denotes the sine-transform of the function $\breve{\chi}$,

$$
\hat{\chi}_{s}(\omega):=\int_{0}^{\infty} \breve{\chi}(\varrho) \sin (\varrho \omega) d \varrho .
$$

Evidently, we have the following imbeddings: $X^{k_{1}} \subset X^{k_{2}}$ and $X_{+}^{k_{1}} \subset$ $X_{+}^{k_{2}}$ for $k_{1}>k_{2}$.

The class $X_{+}^{k}$ is defined in terms of the sine-transform. However the following lemma provides an easily verifiable sufficient condition for nonnegative non-increasing functions to belong to this class (see [9, Lemma 3.2] for proof). 
Lemma A.2. Let $k \geq 1$. If $\chi \in X^{k}, \breve{\chi}(0)=1, \breve{\chi}(\varrho) \geq 0$ for all $\varrho \in(0, \infty)$, and $\breve{\chi}$ is a non-increasing function on $[0,+\infty)$, then $\chi \in X_{+}^{k}$.

The following examples for $\chi$ are presented in [9],

$$
\begin{aligned}
& \chi_{1 k}(x)= \begin{cases}{\left[1-\frac{|x|}{\varepsilon}\right]^{k}} & \text { for } \quad|x|<\varepsilon, \\
0 & \text { for } \quad|x| \geq \varepsilon,\end{cases} \\
& \chi_{2}(x)= \begin{cases}\exp \left[\frac{|x|^{2}}{|x|^{2}-\varepsilon^{2}}\right] & \text { for }|x|<\varepsilon, \\
0 & \text { for }|x| \geq \varepsilon .\end{cases}
\end{aligned}
$$

One can observe that $\chi_{1 k} \in X_{+}^{k}$ for $k \geq 1$, while $\chi_{2} \in X_{+}^{\infty}$ due to Lemma A.2.

\section{Appendix B. Properties of localized potentials}

Here we collect some theorems describing mapping properties of the localized potentials $(2.21),(2.22),(2.23)$, and the corresponding localized boundary operators (2.31) and (2.32).

Note that $\mathcal{V}$ is a weakly singular integral operator (pseudodifferential operator of order -1 ), while $\mathcal{W}$ is a singular integral operator (pseudodifferential operator of order 0 ).

If a localizing function is infinitely differentiable, $\chi \in X^{\infty}$, then the localized potentials and the corresponding boundary operators have the same mapping properties as the corresponding harmonic potentials (see, e.g., [6, Ch. 5, Theorem 2.4(i)], [27], [18, Theorem 8.4.13)]. However, for localizing functions of finite smoothness the localized potential operators may possess quite different properties, in particular, their smoothness is reduced and the smoothness exponents depend on the localizing function (cf. [9]).

Properties of the localized volume potential $\mathcal{P}$ are described in the assertions that follow. Some of them are proved in [9]. We recall that the complete symbol $\mathfrak{S}(\mathbf{P} ; \xi)$ of the operator $\mathbf{P}$ is defined by formula (4.1).

Lemma B.1. (Lemma 5.1 in [9])

(i) Let $\chi \in X^{k}$ with $k \geq 0$. Then $\mathfrak{S}(\mathbf{P} ; \cdot) \in C\left(\mathbb{R}^{3}\right)$,

$$
\mathfrak{S}(\mathbf{P} ; 0)=-\int_{0}^{\infty} \breve{\chi}(\varrho) \varrho d \varrho,
$$

and for $\xi \neq 0$ the following equality holds

$$
\begin{aligned}
\mathfrak{S}(\mathbf{P} ; \xi)= & \sum_{m=0}^{k^{*}} \frac{(-1)^{m+1}}{|\xi|^{2 m+2}} \breve{\chi}^{(2 m)}(0) \\
& -\frac{1}{|\xi|^{k+1}} \int_{0}^{\infty} \sin \left(|\xi| \varrho+\frac{k \pi}{2}\right) \breve{\chi}^{(k)}(\varrho) d \varrho,
\end{aligned}
$$

where $k^{*}$ is the integer part of $(k-1) / 2$ and the sum disappears in (B.1) if $k=0$. 

$\xi \in \mathbb{R}^{3}$.

(ii) If $\chi \in X^{0}$ and condition (A.1) is satisfied, then $\mathfrak{S}(\mathbf{P} ; \xi)<0$ for all

(iii) If $\chi$ has a compact support, then $\mathfrak{S}(\mathbf{P} ; \cdot) \in C^{\infty}\left(\mathbb{R}^{3}\right)$.

Corollary B.2. (Corollary 5.2 in [9])

(i) There exists a positive constant $c_{1}$ such that

$$
|\mathfrak{S}(\mathbf{P} ; \xi)| \leq c_{1}\left(1+|\xi|^{2}\right)^{-\frac{k+1}{2}} \text { for all } \xi \in \mathbb{R}^{3} \quad \text { if } \chi \in X^{k}, k=0,1,
$$

and the operators

$$
\begin{array}{r}
\mathbf{P}: H^{s}\left(\mathbb{R}^{3}\right) \rightarrow H^{s+k+1}\left(\mathbb{R}^{3}\right) \forall s \in \mathbb{R} \quad \text { for } \chi \in X^{k}, \quad k=0,1, \\
: H^{s}\left(\mathbb{R}^{3}\right) \rightarrow H^{s+k+1}\left(\mathbb{R}^{3}\right) \forall s \in \mathbb{R} \\
\text { for } \chi \in X^{k}, \quad k=2,3, \text { and } \chi(0)=0,
\end{array}
$$

are continuous.

(ii) If $\chi \in X_{+}^{1}$, then there exist positive constants $c_{1}$ and $c_{2}$ such that

$$
c_{2}\left(1+|\xi|^{2}\right)^{-1} \leq|\mathfrak{S}(\mathbf{P} ; \xi)| \leq c_{1}\left(1+|\xi|^{2}\right)^{-1} \text { for all } \xi \in \mathbb{R}^{3},
$$

and the operator

$$
\mathbf{P}: H^{s}\left(\mathbb{R}^{3}\right) \rightarrow H^{s+2}\left(\mathbb{R}^{3}\right) \quad \forall s \in \mathbb{R}
$$

is continuously invertible.

For $-1 / 2<r<1 / 2$, let us introduce the following spaces, cf. [24, Definition 3.3],

$$
H^{t, r}\left(\Omega^{ \pm} ; \Delta\right):=\left\{u \in H^{t}\left(\Omega^{ \pm}\right): \Delta u \in H^{r}\left(\Omega^{ \pm}\right)\right\} .
$$

Theorem B.3. (Theorem 5.6 in [9]) If $\chi \in X^{1}$, then the operators

$$
\begin{aligned}
\mathcal{P} & : \widetilde{H}^{s}\left(\Omega^{+}\right) \rightarrow H^{s+2, s}\left(\Omega^{+} ; \Delta\right), \quad-\frac{1}{2}<s<\frac{1}{2}, \\
& : H^{s}\left(\Omega^{+}\right) \rightarrow H^{\frac{5}{2}-\varepsilon, \frac{1}{2}-\varepsilon}\left(\Omega^{+} ; \Delta\right), \quad \frac{1}{2} \leq s, \quad \forall \varepsilon \in(0,1)
\end{aligned}
$$

are continuous.

For $t>1 / 2$, let $\gamma_{S}^{*}$ denote the operator adjoint to the trace operator $\gamma_{S}: H^{t}\left(\mathbb{R}^{3}\right) \rightarrow H^{t-\frac{1}{2}}(\partial \Omega)$, i.e.,

$$
\left\langle\gamma_{S}^{*} v, w\right\rangle_{\mathbb{R}^{3}}:=\left\langle v, \gamma_{S} w\right\rangle_{S} \quad \forall w \in H^{t}\left(\mathbb{R}^{3}\right), \quad v \in H^{\frac{1}{2}-t}(S) .
$$

This definition evidently implies that the operator

$$
\gamma_{S}^{*}: H^{\frac{1}{2}-t}(\partial \Omega) \rightarrow H^{-t}\left(\mathbb{R}^{3}\right), \quad t>1 / 2
$$

is continuous and $\gamma_{S}^{*} v=0$ in $\Omega^{ \pm}$, i.e., $\gamma_{S}^{*} v$ belongs to the space $H_{S}^{-t}$ consisting of distributions from $H^{-t}\left(\mathbb{R}^{3}\right)$ whose supports belong to $S=\partial \Omega$. This also allows us to rewrite the localized single layer potential in terms of the localized volume potential,

$$
\begin{aligned}
& V \psi(y)=-\int_{S} P(x-y) \psi(x) d S_{x}=-\left\langle\gamma_{S} P(\cdot-y), \psi\right\rangle_{S} \\
&=-\left\langle P(\cdot-y), \gamma_{S}^{*} \psi\right\rangle_{\mathbb{R}^{3}}=-\mathbf{P} \gamma_{S}^{*} \psi(y),
\end{aligned}
$$


Note that if the integration surface in (B.5) is $S=\partial \mathbb{R}_{+}^{3}=\left\{x \in R^{3}: x_{3}=0\right\}$, then we have

$$
V \psi(y)=-\mathbf{P}\left(\psi\left(x_{1}, x_{2}\right) \otimes \delta\left(x_{3}\right)\right)(y), \quad y \in \mathbb{R}^{3},
$$

where $\psi\left(x^{\prime}\right) \otimes \delta\left(x_{3}\right) \equiv \gamma_{S}^{*} \psi$ with $x^{\prime}:=\left(x_{1}, x_{2}\right)$ denotes the distributional direct product of the density function $\psi\left(x^{\prime}\right)$ and the Dirac function $\delta\left(x_{3}\right)$. Consequently, for this particular case we can write

$$
\begin{aligned}
& \mathcal{F}_{x \rightarrow \xi}(V \psi(x))(\xi)=-\mathcal{F}_{x \rightarrow \xi}\left(\mathbf{P} \gamma^{*} \psi(x)\right)(\xi) \\
= & -\mathfrak{S}(\mathbf{P})(\xi) \mathcal{F}_{x \rightarrow \xi}\left(\psi\left(x_{1}, x_{2}\right) \otimes \delta\left(x_{3}\right)\right)(\xi)=-\mathfrak{S}(\mathbf{P})(\xi) \mathcal{F}_{x^{\prime} \rightarrow \xi^{\prime}}(\psi)\left(\xi^{\prime}\right) .
\end{aligned}
$$

The localized double layer potential can also be represented in terms of the localized single layer potential,

$$
\begin{aligned}
W \varphi(y) & =-\int_{S}\left[T\left(x, \partial_{x}\right) P(x-y)\right] \varphi(x) d S_{x} \\
& =-\int_{S}\left[a_{k j}(x) n_{k}(x) \partial_{x_{j}} P(x-y)\right] \varphi(x) d S_{x} \\
& =-\partial_{y_{j}} V\left(a_{k j} n_{k} \varphi\right)(y), \quad y \in \mathbb{R}^{3} \backslash S .
\end{aligned}
$$

Now we can prove an analogue of [9, Theorem 5.10] extending it here to a wider range of the exponent $s$ and to the specific double layer potential (B.8).

Theorem B.4. The following operators are continuous

$$
\begin{aligned}
V & : H^{s-\frac{3}{2}}(S) \rightarrow H^{s}\left(\mathbb{R}^{3}\right), \quad s<\frac{3}{2}, \quad \text { for } \chi \in X^{1}, \\
& : H^{s-\frac{3}{2}}(S) \rightarrow H^{s}\left(\Omega^{ \pm}\right), \quad s<k+\frac{1}{2}, \quad \text { for } \chi \in X^{k}, k=1,2, \ldots, \\
& : H^{s-\frac{3}{2}}(S) \rightarrow H^{s, s-1}\left(\Omega^{ \pm} ; \Delta\right), \quad \frac{1}{2}<s<\frac{3}{2}, \quad \text { for } \chi \in X^{2}, \\
W & : H^{s-\frac{1}{2}}(S) \rightarrow H^{s}\left(\Omega^{ \pm}\right), \quad s<k-\frac{1}{2}, \quad \text { for } \quad \chi \in X^{k}, k=1,2, \ldots, \\
& : H^{s-\frac{1}{2}}(S) \rightarrow H^{s, s-1}\left(\Omega^{ \pm} ; \Delta\right), \quad \frac{1}{2}<s<\frac{3}{2}, \quad \text { for } \chi \in X^{3} .
\end{aligned}
$$

Proof. The continuity of operator (B.9) and thus of operator (B.10) for $k=1$ is proved in [9, Theorem 5.10]. To prove continuity of (B.10) for $k=2,3, \ldots$, let us first assume that $\chi$ has a compact support. By Lemma B.1 we can decompose $\mathfrak{S}(\mathbf{P} ; \xi)$ given by $(\mathrm{B} .1)$ as $\mathfrak{S}(\mathbf{P} ; \xi)=\mathfrak{S}_{k \Sigma}(\xi)+\mathfrak{S}_{k I}(\xi)$, where

$$
\begin{aligned}
\mathfrak{S}_{k \Sigma}(\xi):= & \sum_{m=0}^{k^{\prime}} \frac{(-1)^{m+1}}{|\xi|^{2 m+2}} \breve{\chi}^{(2 m)}(0), \\
\mathfrak{S}_{k I}(\xi):= & -\frac{1}{|\xi|^{k+1}} \int_{0}^{\infty} \sin \left(|\xi| \varrho+\frac{k \pi}{2}\right) \breve{\chi}^{(k)}(\varrho) d \varrho \\
& + \begin{cases}\frac{(-1)^{k^{\prime}+1}}{|\xi|^{k+1}} \breve{\chi}^{(k-1)}(0) & \text { if } k \text { is odd } \\
0 & \text { if } k \text { is even }\end{cases}
\end{aligned}
$$


$k^{\prime}$ is the integer part of $(k-2) / 2$ and $\mathfrak{S}_{k \Sigma}(\xi)=0$ if $k=0,1$.

Then $\mathbf{P}=\mathbf{P}_{k \Sigma}+\mathbf{P}_{k I}$, where $\mathbf{P}_{k \Sigma}$ and $\mathbf{P}_{k I}$ are the pseudodifferential operators with the symbols $\mathfrak{S}_{k \Sigma}(\xi)$ and $\mathfrak{S}_{k I}(\xi)$, respectively. Therefore the localized single layer potential with the help of (B.5) can be represented as

$$
V(\psi)=V_{k \Sigma}(\psi)+V_{k I}(\psi)
$$

where $V_{k \Sigma}(\psi)=-\mathbf{P}_{k \Sigma}\left(\gamma_{S}^{*} \psi\right)(y)$ and $V_{k I}(\psi)=-\mathbf{P}_{k I}\left(\gamma_{S}^{*} \psi\right)$.

Note that the symbols $\mathfrak{S}(\mathbf{P})$ and $\mathfrak{S}_{k I}$ are neither of rational type nor classical, in general. Therefore we can not apply directly the well known theorems for pseudodifferential operators with rational type symbols (see, e.g. [6], [18]). However, for $k \geq 2$ the operator $\mathbf{P}_{k \Sigma}$ is of order -2 with the rational symbol $\mathfrak{S}_{k \Sigma}(\xi)$ and we have (see, e.g., Theorem 8.5.8 in [18]),

$$
\begin{aligned}
& V_{k \Sigma}: H^{s-\frac{3}{2}}(S) \rightarrow H^{s}\left(\Omega^{+}\right) \forall s \in \mathbb{R}, \quad \chi \in X^{k}, k=2,3, \ldots, \\
& \mu_{1} V_{k \Sigma}: H^{s-\frac{3}{2}}(S) \rightarrow H^{s}\left(\Omega^{-}\right) \forall s \in \mathbb{R}, \\
& \forall \mu_{1} \in \mathcal{D}\left(\mathbb{R}^{3}\right), \quad \chi \in X^{k}, \quad k=2,3, \ldots
\end{aligned}
$$

On the other hand, we can write

$$
\mathfrak{S}_{k I}(\xi):=\lambda(\xi) \mathfrak{S}_{k I}(\xi)+[1-\lambda(\xi)] \mathfrak{S}_{k I}(\xi),
$$

where $\lambda \in \mathcal{D}\left(\mathbb{R}^{3}\right)$ is a cut-off function such that $\lambda(\xi)=1$ for $|\xi| \leq 1$. The first summand in (B.19) has a compact support and defines a pseudodifferential operator which maps $H^{s}\left(\mathbb{R}^{3}\right)$ into $C^{\infty}\left(\mathbb{R}^{3}\right)$ for arbitrary $s \in \mathbb{R}$. By (B.15) and Definition A.1 of the classes $X^{k}$, we have $\mathfrak{S}_{k I}(\xi)=\mathcal{O}\left(|\xi|^{-k-1}\right)$ for sufficiently large $|\xi|$, implying that the second summand in (B.19) defines a pseudodifferential operator of order $-(k+1)$. Thus we obtain continuity of the operator

$$
\begin{aligned}
& \mu_{1} \mathbf{P}_{k I}: H^{s-k-1}\left(\mathbb{R}^{3}\right) \rightarrow H^{s}\left(\mathbb{R}^{3}\right) \\
& \text { for } s \in \mathbb{R}, \quad \chi \in X^{k}, \quad \forall \mu_{1} \in \mathcal{D}\left(\mathbb{R}^{3}\right), \quad k=0,1,2, \ldots
\end{aligned}
$$

Then by (B.4) we have the continuity of the operator

$$
\begin{aligned}
& \mu_{1} \mathbf{P}_{k I} \gamma^{*}: H^{s-k-\frac{1}{2}}(S) \rightarrow H^{s}\left(\mathbb{R}^{3}\right) \\
& \text { for } s<k+\frac{1}{2}, \chi \in X^{k}, \forall \mu_{1} \in \mathcal{D}\left(\mathbb{R}^{3}\right), k=0,1,2, \ldots
\end{aligned}
$$

Hence (B.16), (B.17), and (B.20) imply (B.10) for the bounded domain $\Omega^{+}$, while (B.16), (B.18), and (B.20) imply continuity of the operator

$$
\begin{aligned}
& \mu_{1} V: H^{s-\frac{3}{2}}(S) \rightarrow H^{s}\left(\Omega^{-}\right) \\
& \text {for } s<k+\frac{1}{2}, \chi \in X^{k}, \forall \mu_{1} \in \mathcal{D}\left(\mathbb{R}^{3}\right), \quad k=2,3, \ldots
\end{aligned}
$$

for the unbounded domain $\Omega^{-}$. Choosing $\mu_{1}$ such that $\mu_{1}=1$ on the compact support of $V$ (defined only by $S$ and by the compact support of $\chi$ ), we obtain that $\mu_{1} V=V$, i.e., $\mu_{1}$ can be dropped in (B.21) implying (B.10) also for $\Omega^{-}$.

Let now $\chi$ be not compactly supported. Let us introduce a radial function $\mu_{0} \in \mathcal{D}\left(\mathbb{R}^{3}\right)$ with respect to the origin of the coordinate system such 
that $\mu_{0}(x)=1$ in a neighbourhood of 0 and represent $\chi=\chi_{0}+\chi_{\infty}$ with $\chi_{0}=\mu_{0} \chi$ and $\chi_{\infty}=\left(1-\mu_{0}\right) \chi$. Evidently,

$$
V \psi=V_{\chi_{0}} \psi+V_{\chi_{\infty}} \psi
$$

For $\chi \in X^{k}$ we see that $\chi_{0} \in X^{k}$ and is compactly supported, implying (B.10) for $V_{\chi_{0}}$. On the other hand, $\chi_{\infty} \in X^{k}$ and $\breve{\chi}_{\infty}^{(q)}(0)=0, q=0,1,2, \ldots$ Then by Lemma B.1 applied to $\chi_{\infty}$ we obtain the following estimate for the corresponding symbol

$$
\left|\mathfrak{S}\left(\mathbf{P}_{\chi_{\infty}} ; \xi\right)\right| \leq c_{1}\left(1+|\xi|^{2}\right)^{-\frac{k+1}{2}} \text { for all } \xi \in \mathbb{R}^{3} \quad \text { if } \chi \in X^{k}, k=0,1,2, \ldots
$$

Therefore the volume potential

$$
\mathbf{P}_{\chi_{\infty}}: H^{s-k-1}\left(\mathbb{R}^{3}\right) \rightarrow H^{s}\left(\mathbb{R}^{3}\right) \text { for } s \in \mathbb{R}, \quad \chi \in X^{k}, \quad k=0,1,2, \ldots,
$$

and thus the single layer potential

$$
V_{\chi_{\infty}}: H^{s-k-\frac{1}{2}}(\partial \Omega) \rightarrow H^{s}\left(\mathbb{R}^{3}\right), \quad s<k+\frac{1}{2}, \quad \chi \in X^{k}, \quad k=0,1,2, \ldots
$$

are continuous, which implies (B.10) with non-compactly supported $\chi$.

Mapping (B.10) can be evidently rewritten as

$$
V: H^{s-\frac{1}{2}}(S) \rightarrow H^{s+1}\left(\Omega^{ \pm}\right) \text {for } s<k-\frac{1}{2}, \quad \chi \in X^{k}, \quad k=1,2, \ldots
$$

Then (B.8) implies the continuity of operators (B.12).

The continuity of (B.11) is already proved in [9, Theorem 5.10]. To prove continuity of (B.13), we first remark that $\mathfrak{S}_{2 \Sigma}=\mathfrak{S}_{3 \Sigma}=-1 /|\xi|^{2}$ by (B.14) since $\breve{\chi}(0)=1$, which implies $V_{2 \Sigma} \psi=V_{3 \Sigma} \psi$ is the classical (nonlocalised) single layer potential harmonic in $\Omega^{ \pm}$. Then by (B.16) we obtain $r_{\Omega^{ \pm}} \Delta V \psi=-r_{\Omega^{ \pm}} \Delta \mathbf{P}_{k I}\left(\gamma_{S}^{*} \psi\right)$ for $k=2,3$. Rewriting operator (B.20) as

$$
\begin{aligned}
& \mu_{1} \mathbf{P}_{k I} \gamma^{*}: H^{s-\frac{3}{2}}(S) \rightarrow H^{s+k-1}\left(\mathbb{R}^{3}\right) \\
& \text { for } s<\frac{3}{2}, \quad \chi \in X^{k}, \quad \forall \mu_{1} \in \mathcal{D}\left(\mathbb{R}^{3}\right), \quad k=0,1,2, \ldots,
\end{aligned}
$$

and applying the Laplace operator, we obtain the continuity of the operator

$$
\begin{aligned}
\mu_{1} \Delta V: H^{s-\frac{3}{2}}(S) \rightarrow & H^{s+k-3}\left(\Omega^{ \pm}\right) \\
\qquad & \text { for } s<\frac{3}{2}, \chi \in X^{k}, \forall \mu_{1} \in \mathcal{D}\left(\mathbb{R}^{3}\right), k=2,3 .
\end{aligned}
$$

The multiplier $\mu_{1}$ can be evidently dropped in (B.24) for the bounded domain $\Omega^{+}$. It can be dropped also for the unbounded domain $\Omega^{-}$if $\chi$ has a compact support. If $\chi$ is not compactly supported, we again represent $V$ as in (B.22) and, taking into account (B.23), we finally conclude that $\mu_{1}$ can be dropped in (B.25) also for the non-compactly supported $\chi$, and we have

$$
\Delta V: H^{s-\frac{3}{2}}(S) \rightarrow H^{s+k-3}\left(\Omega^{ \pm}\right) \quad \text { for } \quad s<\frac{3}{2}, \chi \in X^{k}, k=2,3 .
$$

Along with (B.10) this particularly implies (B.11) if we take $k=2$. 
Applying the Laplace operator to (B.8) and taking into account (B.25), we obtain

$$
\Delta W: H^{s-\frac{3}{2}}(S) \rightarrow H^{s+k-4}\left(\Omega^{ \pm}\right) \text {for } s<\frac{3}{2}, \quad k=2,3 .
$$

For $k=3$, in view of (B.12) this particularly implies (B.13).

Theorem B.5. The following jump relations hold on $S$ :

$$
\begin{array}{r}
\gamma^{ \pm} V \psi=\mathcal{V} \psi, \quad \psi \in H^{s-\frac{3}{2}}(S), \quad \frac{1}{2}<s<k+\frac{1}{2} \\
\text { for } \quad \chi \in X^{k}, \quad k=1,2, \ldots, \\
\gamma^{ \pm} W \varphi=\mp \mu \varphi+\mathcal{W} \varphi, \quad \varphi \in H^{s-\frac{1}{2}}(S), \quad \frac{1}{2}<s<k-\frac{1}{2} \\
\text { for } \quad \chi \in X^{k}, \quad k=2,3, \ldots,
\end{array}
$$

where $\mu(y)$ is given by (2.33) and the operators

$$
\begin{gathered}
\mathcal{V}: H^{s-\frac{3}{2}}(S) \rightarrow H^{s-\frac{1}{2}}(S), \quad \frac{1}{2}<s<k+\frac{1}{2} \quad \text { for } \chi \in X^{k}, \quad k=1,2, \ldots, \\
\mathcal{W}: H^{s-\frac{1}{2}}(S) \rightarrow H^{s-\frac{1}{2}}(S), \quad \frac{1}{2}<s<k-\frac{1}{2} \quad \text { for } \chi \in X^{k}, \quad k=2,3, \ldots,
\end{gathered}
$$

are continuous.

Proof. Relation (B.26) was proved in [9, Theorem 5.13] for $k=1$. For a smooth function $\psi$ the following jump relations for derivatives of the single layer potential hold on $S$ (cf. [27], Ch. 2, Section 14, formula (14.12))

$$
\left\{\partial_{y_{j}} V \psi\right\}^{ \pm}= \pm \frac{1}{2} n_{j} \psi(y)-\int_{S} \partial_{y_{j}} P(x-y) \psi(x) d S_{x}, \quad y \in S, \quad \chi \in X^{2} .
$$

The singular kernel $\partial_{y_{j}} P(x-y)$ satisfies the Tricomi condition, cf. [26, Ch.IX, Section 1, Theorem 1.1], [18, condition (7.1.17) and Theorem 7.1.7], and the surface integral in (B.30) is understood in the Cauchy principal value sense. Moreover, operators (B.30) can be treated as pseudodifferential operators of order zero ([1], [2], [15], [18, Chapters 7-8]). Substituting (B.30) in (B.8) we obtain (B.27) for smooth functions $\psi$. Taking into account the mapping properties of the operators $V$ and $W$ given by Theorem B.4, these relations can be extended then by continuity to the appropriate spaces, giving also the continuity of (B.28)-(B.29) (cf. [9], [18, Theorem 8.6.3 and Section 9.2]).

Lemma B.6. [9, Lemma 6.3] Let $\chi \in X_{+}^{1}, \psi \in H^{-\frac{1}{2}}(\partial \Omega)$, and $f \in H^{0}\left(\Omega^{+}\right)$. If $V \psi+\mathcal{P} f=0$ in $\Omega^{+}$, then $\psi=0$ on $\partial \Omega$ and $f=0$ in $\Omega^{+}$.

Remark B.7. The principal homogeneous symbols of the pseudodifferential operators $\mathcal{V}$ and $-\mu \mathbf{I}+\mathcal{W}$ calculated in a local coordinate system with the 
origin at a point $\widetilde{y} \in S$ and the third axis coinciding with the outward unit normal vector $n(\widetilde{y})$ to $S$ at the point $\widetilde{y}$, read as (cf., [7], [8], [18])

$$
\begin{aligned}
\mathfrak{S}_{0}(\mathcal{V})\left(\widetilde{y}, \xi^{\prime}\right) \equiv \mathfrak{S}_{0}(\widetilde{\mathcal{V}}) & \left(\widetilde{y}, \xi^{\prime}\right)=\frac{1}{2\left|\xi^{\prime}\right|}, \quad \xi^{\prime} \in \mathbb{R}^{2} \backslash\{0\}, \\
\mathfrak{S}_{0}(-\mu \mathbf{I}+\mathcal{W})\left(\widetilde{y}, \xi^{\prime}\right) & \equiv \mathfrak{S}_{0}(-\mu \mathbf{I}+\widetilde{\mathcal{W}})\left(\widetilde{y}, \xi^{\prime}\right) \\
& =-\frac{1}{2} \widetilde{a}_{33}(\widetilde{y})-\frac{i}{2} \sum_{l=1}^{2} \widetilde{a}_{3 l}(\widetilde{y}) \frac{\xi_{l}}{\left|\xi^{\prime}\right|} .
\end{aligned}
$$

Here $\widetilde{a}_{k j}(\widetilde{y})$ is given by $(5.11)$, where $\Lambda(\widetilde{y})=\left[\lambda_{k j}(\widetilde{y})\right]_{3 \times 3}$ is an orthogonal matrix with the property $\Lambda^{\top} n(\widetilde{y})=(0,0,-1)^{\top}$. Therefore $\lambda_{p 3}(\widetilde{y})=-n_{p}(\widetilde{y})$, $p=1,2,3$. In view of $(2.33)$ it is evident that

$$
\frac{1}{2} \widetilde{a}_{33}(\widetilde{y})=\frac{1}{2} \lambda_{p 3}(\widetilde{y}) a_{p q}(\widetilde{y}) \lambda_{q 3}(\widetilde{y})=\mu(\widetilde{y})>0 .
$$

\section{Appendix C. Representation for $\Pi^{\prime}$}

Here we derive representation (5.7) for the operator $\Pi^{\prime}$ defined by (5.5), i.e., we show that

$$
\begin{aligned}
\Pi^{\prime}(g)\left(\xi^{\prime}\right) & =\lim _{x_{3} \rightarrow 0+} r_{\mathbb{R}_{+}} \mathcal{F}_{\xi_{3} \rightarrow x_{3}}^{-1}\left[g\left(\xi^{\prime}, \xi_{3}\right)\right] \\
& =-\frac{1}{2 \pi} \int_{\Gamma^{-}} g\left(\xi^{\prime}, \zeta\right) d \zeta, \quad \xi^{\prime} \in \mathbb{R}^{2} \backslash\{0\},
\end{aligned}
$$

if the following conditions hold:

(i) $g\left(\xi^{\prime}, \xi_{3}\right)$ is rational in $\xi_{3}$ and the denominator does not vanish for nonzero real $\xi=\left(\xi^{\prime}, \xi_{3}\right) \in \mathbb{R}^{3} \backslash\{0\}$,

(ii) $g\left(\xi^{\prime}, \xi_{3}\right)$ is homogeneous of order $m \in \mathbb{Z}:=\{0, \pm 1, \pm 2, \ldots\}$ in $\xi=$ $\left(\xi^{\prime}, \xi_{3}\right)$, and

(iii) $g\left(\xi^{\prime}, \xi_{3}\right)$ is infinitely differentiable with respect to real $\xi=\left(\xi^{\prime}, \xi_{3}\right)$ for $\xi^{\prime} \neq 0$.

Then it follows that the function $g^{*}\left(\omega, r, \xi_{3}\right):=g\left(\xi^{\prime}, \xi_{3}\right) \equiv g\left(r \omega, \xi_{3}\right)$ with $r=\left|\xi^{\prime}\right|$ and $\omega=\xi^{\prime} /\left|\xi^{\prime}\right|$ is infinitely differentiable with respect to the real variables $\omega, r$, and $\xi_{3}$ for $|\omega|=1, r \in[0,+\infty), \xi_{3} \in(-\infty,+\infty)$ and $r+\left|\xi_{3}\right|>0$. Moreover,

$$
\begin{aligned}
& g(0,0,-1)=e^{-i m \pi} g(0,0,+1), \\
& \frac{\partial^{k} g^{*}(\omega, 0,-1)}{\partial r^{k}}=(-1)^{k} e^{-i m \pi} \frac{\partial^{k} g^{*}(\omega, 0,+1)}{\partial r^{k}}, \quad k=1,2, \ldots
\end{aligned}
$$

Therefore such a function $g$ belongs to the class of symbols $D_{m}$ introduced in [15, Ch. 3, Sections 3.10].

By Lemma 10.1 in [15] we deduce that $g$ admits the decomposition

$$
g\left(\xi^{\prime}, \xi_{3}\right)=g^{-}\left(\xi^{\prime}, \xi_{3}\right)+R_{N}\left(\xi^{\prime}, \xi_{3}\right),
$$


where $g^{-}\left(\xi^{\prime}, \xi_{3}\right)$ is a rational function in $\xi_{3}$ and has an analytic continuation $g^{-}\left(\xi^{\prime}, \xi_{3}+i \tau\right)$ with respect to $\zeta=\xi_{3}+i \tau$ into the lower complex half-plane $\tau<0$, which is continuous for $\left|\xi^{\prime}\right|+\left|\xi_{3}\right|+|\tau| \neq 0$ and satisfies the estimate

$$
\left|g^{-}\left(\xi^{\prime}, \xi_{3}+i \tau\right)\right| \leqslant c_{N}^{\prime}\left(\left|\xi^{\prime}\right|+\left|\xi_{3}\right|+|\tau|\right)^{m},
$$

while $R_{N}\left(\xi^{\prime}, \xi_{3}\right)$ is a homogeneous function of degree $m$ satisfying the estimate

$$
\left|R_{N}\left(\xi^{\prime}, \xi_{3}\right)\right| \leqslant c_{N}^{\prime \prime}\left|\xi^{\prime}\right|^{N+1}\left(\left|\xi^{\prime}\right|+\left|\xi_{3}\right|\right)^{m-N-1}
$$

with arbitrary natural number $N$ and some positive constants $c_{N}^{\prime}$ and $c_{N}^{\prime \prime}$. In view of (C.2), $R_{N}\left(\xi^{\prime}, \xi_{3}\right)$ is rational in $\xi_{3}$ and the poles of the functions $g\left(\xi^{\prime}, \zeta\right)$ and $R_{N}\left(\xi^{\prime}, \zeta\right)$ in the lower complex half-plane coincide.

It can be easily shown that for $\tau<0, \xi^{\prime} \neq 0$, and $2 m+2 s+1<0$ the estimate (C.3) implies

$$
\begin{aligned}
& \int_{-\infty}^{+\infty}\left(1+\left|\xi_{3}\right|+|\tau|\right)^{2 s}\left|g^{-}\left(\xi^{\prime}, \xi_{3}+i \tau\right)\right|^{2} d \xi_{3} \\
& \quad \leqslant c_{N}^{\prime} \int_{-\infty}^{+\infty}\left(1+\left|\xi_{3}\right|+|\tau|\right)^{2 s}\left(\left|\xi^{\prime}\right|+\left|\xi_{3}\right|+|\tau|\right)^{2 m} d \xi_{3} \leqslant C_{N}\left(1+\left|\xi^{\prime}\right|^{2 m}\right),
\end{aligned}
$$

where the positive constant $C_{N}$ does not depend neither on $\xi^{\prime}$ nor on $\tau$.

Now, in accordance with the Paley-Wiener theorem in one dimensional case (obtained e.g. from [15, Theorem 4.5], where the dependence on and integration over $\xi^{\prime}$ should be omitted), for any $s<-m-1 / 2$ and any fixed $\xi^{\prime} \neq 0$ there is a function $f\left(\xi^{\prime}, \cdot\right) \in \widetilde{H}^{s}\left(\mathbb{R}_{-}\right)$such that

$$
g^{-}\left(\xi^{\prime}, \xi_{3}+i \tau\right)=\mathcal{F}_{x_{3} \rightarrow \xi_{3}}\left[f\left(\xi^{\prime}, x_{3}\right) e^{-x_{3} \tau}\right], \quad \tau<0 .
$$

Hence, the equation

$$
\mathcal{F}_{\xi_{3} \rightarrow x_{3}}^{-1}\left[g^{-}\left(\xi^{\prime}, \xi_{3}+i \tau\right)\right]=f\left(\xi^{\prime}, x_{3}\right) e^{-x_{3} \tau}=0 \quad \text { for } x_{3}>0
$$

follows with arbitrary $\tau<0$, since $f\left(\xi^{\prime}, \cdot\right) \in \widetilde{H}^{s}\left(\mathbb{R}_{-}\right)$.

Taking into account that $g^{-}\left(\xi^{\prime}, \xi_{3}+i \tau\right)$ is holomorphic in the lower half-plane $\tau<0$ and continuous for $\left|\xi^{\prime}\right|+\left|\xi_{3}\right|+|\tau| \neq 0$, and since the direct and inverse Fourier transform operators are continuous in appropriate function spaces, we deduce from (C.5) by passing to the limit as $\tau \rightarrow 0-$, that $\mathcal{F}_{\xi_{3} \rightarrow x_{3}}^{-1}\left[g^{-}\left(\xi^{\prime}, \xi_{3}\right)\right]=0$ for $x_{3}>0$. Therefore,

$$
\lim _{x_{3} \rightarrow 0+} r_{\mathbb{R}_{+}} \mathcal{F}_{\xi_{3} \rightarrow x_{3}}^{-1}\left[g^{-}\left(\xi^{\prime}, \xi_{3}\right)\right] \equiv \Pi^{\prime}\left(g^{-}\right)\left(\xi^{\prime}\right)=0 \quad \forall \xi^{\prime} \in \mathbb{R}^{2} \backslash\{0\} .
$$

Further, with the help of decomposition (C.2) with $N>m+1$ and using the estimate (C.4) for $\xi^{\prime} \neq 0$, we see that $R_{N}\left(\xi^{\prime}, \cdot\right) \in L_{1}(\mathbb{R})$. With the help of (C.6) we get from (C.1)

$$
\Pi^{\prime}(g)\left(\xi^{\prime}\right)=\Pi^{\prime}\left(R_{N}\right)\left(\xi^{\prime}\right)=\frac{1}{2 \pi} \int_{-\infty}^{+\infty} R_{N}\left(\xi^{\prime}, \xi_{3}\right) d \xi_{3}=-\frac{1}{2 \pi} \int_{\Gamma^{-}} R_{N}\left(\xi^{\prime}, \zeta\right) d \zeta
$$

where $\Gamma^{-}$is a contour in the lower complex half-plane orientated anticlockwise and enclosing all the poles of the rational function $R_{N}\left(\xi^{\prime}, \zeta\right)$ in the lower complex half-plane. 
Finally, due to relation (C.2) and since $g^{-}\left(\xi^{\prime}, \zeta\right)$ is holomorphic in the lower complex half-plane, we arrive at the equality

$$
\Pi^{\prime}(g)\left(\xi^{\prime}\right)=-\frac{1}{2 \pi} \int_{\Gamma^{-}}\left[g^{-}\left(\xi^{\prime}, \zeta\right)+R_{N}\left(\xi^{\prime}, \zeta\right)\right] d \zeta=-\frac{1}{2 \pi} \int_{\Gamma^{-}} g\left(\xi^{\prime}, \zeta\right) d \zeta,
$$

which completes the proof of relation (C.1).

\section{Acknowledgment}

This research was supported by the grant EP/H020497/1: "Mathematical Analysis of Localized Boundary-Domain Integral Equations for VariableCoefficient Boundary Value Problems" from the EPSRC, UK.

\section{References}

[1] M.S. Agranovich, Elliptic singular integro-differential operators. Uspekhi Mat. Nauk, 20 (1965), No. 5, 3-120.

[2] M.S. Agranovich, N.N. Voitovich, B.Z. Katsenelenbaum, A.N. Sivov, Generalized method of eigenoscillations in diffraction theory. Wiley, Berlin, 1999.

[3] T.G. Ayele, S.E. Mikhailov, Two-operator boundary-domain integral equations for a variable-coefficient BVP. In: Integral Methods in Science and Engineering (edited by C. Constanda, M. Pérez), Vol. 1: Analytic Methods, Birkhäuser, Boston-Basel-Berlin, 2010, ISBN 978-08176-4898-5, 29-39.

[4] T.G. Ayele, S.E. Mikhailov, Analysis of two-operator boundary-domain integral equations for a variable-coefficient mixed BVP. Eurasian Mathematical Journal, 2 (2011), No 3, 20-41.

[5] L. Boutet de Monvel, Boundary problems for pseudo-differential operators. Acta Math., 126 (1971), 11-51.

[6] J. Chazarain and A. Piriou, Introduction to the Theory of Linear Partial Differential Equations. North-Holland, Amsterdam, 1982.

[7] O. Chkadua, S. Mikhailov, D. Natroshvili, Analysis of direct boundary-domain integral equations for a mixed BVP with variable coefficient. I: Equivalence and invertibility. J. Integral Equations Appl., 21 (2009), 499-542.

[8] O. Chkadua, S. Mikhailov, D. Natroshvili, Analysis of direct boundary-domain integral equations for a mixed BVP with variable coefficient. II: Solution regularity and asymptotics. J. Integral Equations Appl., 22 (2010), 19-37.

[9] O. Chkadua, S.E. Mikhailov, D. Natroshvili, Analysis of some localized boundary-domain integral equations. J. Integral Equations Appl., 21 (2009), 407-447.

[10] O. Chkadua, S. E. Mikhailov, D. Natroshvili, Analysis of segregated boundarydomain integral equations for variable-coefficient problems with cracks. Numerical Methods for Partial Differential Equations, 27 (2011), 121-140.

[11] O. Chkadua, S. E. Mikhailov, D. Natroshvili, Analysis of some localised boundary-domain integral equations for transmission problems with variable coefficients. In: Integral Methods in Science and Engineering (edited by C. Constanda and P. Harris), Birkhäuser, Boston, 2011, ISBN 978-0-8176-8237-8, 91-108. 
[12] O. Chkadua, S. E. Mikhailov, D. Natroshvili, Localized direct segregated boundary-domain integral equations for variable-coefficient transmission problems with interface crack. Memoirs on Differential Equations and Mathematical Physics, 52 (2011), 17-64.

[13] O. Chkadua, S. E. Mikhailov, D. Natroshvili, Analysis of direct segregated boundary-domain integral equations for variable-coefficient mixed BVPs in exterior domains, Analysis and Applications, 11 (2013), DOI: 10.1142/S0219530513500061 (to appear).

[14] M. Costabel, Boundary integral operators on Lipschitz domains: elementary results. SIAM J. Math. Anal., 19 (1988), 613-626.

[15] G. Eskin, Boundary Value Problems for Elliptic Pseudodifferential Equations. Transl. of Mathem. Monographs, Amer. Math. Soc., 52, Providence, Rhode Island, 1981. [Russian original: Nauka publishing, Moscow, 1973.]

[16] P. Grisvard, Elliptic Problems in Nonsmooth Domains. Pitman, BostonLondon-Melbourne, 1985.

[17] G. Grubb, Distributions and operators, Springer, New York, 2009.

[18] G.C. Hsiao, W.L. Wendland, Boundary Integral Equations. Springer, BerlinHeidelberg, 2008.

[19] J.-L. Lions, E. Magenes, Non-homogeneous boundary value problems and applications. Vol. I. Springer, New York-Heidelberg, 1972.

[20] W. McLean, Strongly Elliptic Systems and Boundary Integral Equations. Cambridge University Press, Cambridge, UK, 2000.

[21] S.E. Mikhailov, Localized boundary-domain integral formulation for problems with variable coefficients. Int. J. Engineering Analysis with Boundary Elements, 26 (2002), 681-690.

[22] S.E. Mikhailov, Localized direct boundary-domain integro-differential formulations for scalar nonlinear boundary-value problems with variable coefficients. J. Engineering Math., 51 (2005), 283-302.

[23] S.E. Mikhailov, Analysis of united boundary-domain integro-differential and integral equations for a mixed BVP with variable coefficient. Math. Methods Appl. Sci., 29 (2006), 715-739.

[24] S.E. Mikhailov, Traces, extensions and co-normal derivatives for elliptic systems on Lipschitz domains. J. Math. Analysis and Appl., 378, 324-342 (2011)

[25] S.E. Mikhailov, I.S. Nakhova, Mesh-based numerical implementation of the localized boundary-domain integral equation method to a variable-coefficient $\mathrm{Neu}$ mann problem, J. Engineering Math., 51 (2005) 251-259.

[26] S.G. Mikhlin, S. Prössdorf, Singular Integral Operators. Springer, Berlin, 1986.

[27] C. Miranda, Partial differential equations of elliptic type. Springer, New YorkBerlin, 1970.

[28] S. Rempel and B.-W. Schulze, Index theory of elliptic boundary problems. Akademie-Verlag, Berlin, 1982.

[29] E. Shargorodsky, An $\mathbb{L}_{p}$-Analogue of the Vishik-Eskin Theory. Mem. Diff. Equations Math. Phys., 2 (1994), 41-146.

[30] J. Sladek, V. Sladek, S.N. Atluri, Local boundary integral equation (LBIE) method for solving problems of elasticity with nonhomogeneous material properties. Comput. Mech., 24 (2000), No. 6, 456-462. 
[31] A.E. Taigbenu, The Green element method. Kluwer, 1999.

[32] T. Zhu, J.-D. Zhang, S. N. Atluri, A local boundary integral equation (LBIE) method in computational mechanics, and a meshless discretization approach. Comput. Mech., 21 (1998), 223-235.

[33] T. Zhu, J.-D. Zhang, S.N. Atluri, A meshless numerical method based on the local boundary integral equation (LBIE) to solve linear and non-linear boundary value problems. Eng. Anal. Bound. Elem., 23 (1999), 375-389.

O. Chkadua

I.Javakhishvili Tbilisi State University, A.Razmadze Mathematical Institute,

2, University st., Tbilisi 0186, Georgia

\& Sokhumi State University,

9, Politkovskaia str., Tbilisi 0186, Georgia

e-mail: chkadua@rmi.ge

S.E. Mikhailov

Department of Mathematics, Brunel University London,

Uxbridge, UB8 3PH, UK

e-mail: sergey.mikhailov@brunel.ac.uk

D. Natroshvili

Dept. of Mathematics, Georgian Technical University,

77, M.Kostava str., Tbilisi 0175, Georgia

\& I. Vekua Institute of Applied Mathematics, Tbilisi State University,

2, University str., Tbilisi 0186, Georgia

e-mail: natrosh@hotmail.com 